Comparing the lipid membrane affinity and permeation of drug-like acids

The intriguing effects of cholesterol and charged lipids

\author{
Journal Article
}

Author(s):

Thomae, Anita V.; Koch, Tamara; Panse, Christian; Wunderli-Allenspach, Heidi; Krämer, Stefanie-Dorothea (i)

Publication date:

2007

Permanent link:

https://doi.org/10.3929/ethz-b-000003333

Rights / license:

In Copyright - Non-Commercial Use Permitted

Originally published in:

Pharmaceutical Research 24(8), https://doi.org/10.1007/s11095-007-9263-y 


\title{
Comparing the Lipid Membrane Affinity and Permeation of Drug-like Acids: The Intriguing Effects of Cholesterol and Charged Lipids
}

\author{
Anita V. Thomae, ${ }^{1}$ Tamara Koch, ${ }^{1}$ Christian Panse, ${ }^{2}$ Heidi Wunderli-Allenspach, ${ }^{1}$ and Stefanie D. Krämer ${ }^{1,3}$
}

Received December 5, 2006; accepted February 6, 2007; published online March 27, 2007

\begin{abstract}
Purpose. Lipid bilayers regulate the passage of solutes into and between cellular compartments. A general prerequisite for this passage is the partitioning of the solute into the bilayer. We investigated the relationship between bilayer partitioning and permeation of three drug-like acids in liposomal systems consisting of phosphatidylcholine alone or mixed with cholesterol or charged lipids.

Materials and Methods. Bilayer partitioning was determined by equilibrium dialysis. Bilayer permeation was studied with a luminescence assay which is based on the energy transfer of the permeant to intraliposomal terbium(III).

Results. The influence of the lipid composition on the pH-dependent membrane affinity was in accordance with the membrane rigidity and possible electrostatic interactions between the acids and the lipids. However, there was no direct relationship between membrane affinity and permeation. This seeming discrepancy was closer analyzed with numerical simulations of the permeation process based on the single rate constants for partitioning and translocation. The simulations were in line with our experimental findings.

Conclusions. Depending on the single rate constants and on the geometry of the system, lipid bilayer permeation may positively, negatively or not correlate with the bilayer affinity of the permeant.
\end{abstract}

KEY WORDS: cholesterol; lipid bilayer; liposome; membrane affinity; membrane permeation.

\section{INTRODUCTION}

Lipid bilayers constitute the physical barriers which restrict the free passage of solutes into and between the intracellular and extracellular compartments of the animal and human body. Lipophilic compounds can overcome these barriers by passive permeation according to their physicochemical properties. This is of high relevance in drug therapy as most therapeutic agents need to cross in vivo barriers to reach their targets. Lipid bilayer permeation of drug-like lipophilic molecules is a complex process that involves (1) association of the solute to the outer lipid leaflet of the membrane, (2) translocation (flip-flop or diffusion) of the solute to

Electronic supplementary material. The online version of this article (doi: 10.1007/s11095-007-9263-y) contains supplementary material, which is available to authorized users.

\footnotetext{
${ }^{1}$ Institute of Pharmaceutical Sciences, Department of Chemistry and Applied Biosciences, ETH Zurich, Wolfgang-Pauli-Strasse 10, CH8093 Zurich, Switzerland.

${ }^{2}$ Functional Genomics Center, UNI/ETH Zurich, Zurich, Switzerland.

${ }^{3}$ To whom correspondence should be addressed. (e-mail: skraemer@ pharma.ethz.ch)

ABBREVIATIONS: AA, Anthranilic acid; ACA, aromatic carboxylic acid; Chol, Cholesterol; DCBA, 3,5-Dichlorbenzoic acid; PhC, Phosphatidylcholine; PhI, Phosphatidylinositol; SA, Salicylic acid; StAm, Stearylamine; 2-OH-NA, 2-Hydroxynicotinic acid.
}

the inner lipid leaflet and (3) dissociation of the solute from the inner lipid leaflet (1-3). Based on this model, permeation is determined by two equilibria: the partitioning between the aqueous compartments and the lipid leaflets with the partition coefficient as the equilibrium constant and the translocation (or diffusion) between the two lipid leaflets.

According to this model the membrane partitioning of a drug-like compound has an influence on permeation but the two processes must not necessarily correlate as the translocation from one leaflet to the other may be rate limiting. This is discussed e.g. by Mayer et al. (4) with regard to peptide permeation.

Several studies have shown that the lipid composition has a major impact on the membrane permeation of drugs (5-7). This could result from the influence of the lipid composition on the membrane affinity of the solutes $(6,8)$, from its influence on the translocation or from a combination of both. A detailed analysis and comparison of the influence of the lipid composition on the $\mathrm{pH}$-dependent membrane affinity and permeation of a compound should extend our understanding of the permeation process.

The $\mathrm{pH}$-partition hypothesis is generally applied to estimate membrane permeation at a particular $\mathrm{pH}$, assuming that only the neutral form of an acid or a base can permeate lipid bilayers (9-13). Contradicting the pH-partition hypothesis, we demonstrated recently with a liposomal permeation assay that the anionic species of small aromatic carboxylic acids (ACAs) permeated egg phosphatidylcholine ( $\mathrm{PhC})$ 
bilayers only 12-155 times slower than the net neutral species did and controlled permeation at neutral pH 7 (14).

Here, we used the same permeation assay, which monitors the changes in luminescence of intraliposomal $\mathrm{Tb}^{3+}$ upon ligation with permeated ACAs, in combination with membrane affinity measurements by equilibrium dialysis, to investigate the influence of cholesterol (Chol) and the charged lipids phosphatidylinositol (PhI) and stearylamine (StAm) on the membrane affinity and the membrane permeation of the ACAs (Table I) salicylic acid (SA), anthranilic acid (AA) and 2-hydroxynicotinic acid (2OH-NA). As these acids all have the possibility to form intramolecular hydrogen bonds, we investigated $\mathrm{PhC}$ bilayer permeation of 3,5-dichlorobenzoic acid (DCBA), in addition. The latter lacks the possibility of strong intramolecular hydrogen bonding.

$\mathrm{PhC}$, which is the most abundant phospholipid in mammalian cell membranes, was used as reference lipid. Chol is present in mammalian cell membranes in various amounts up to about $50 \%$. It is known to influence membrane fluidity (15-17) and to affect the membrane affinity and permeation of solutes (17). The influence of the negatively charged $\mathrm{PhI}$ and of the positively charged StAm was analysed to elucidate to which extent electrostatic interactions affect the permeation process and the membrane affinity.

With this study, it could be demonstrated that both membrane affinity and membrane permeation are influenced by the membrane composition. The membrane affinity of the three test compounds is similarly affected by the investigated lipids. However, the influence of the lipid composition on the membrane permeation is not consistent among the tested solutes. The membrane composition can have opposite effects on compound partitioning and permeation. There was no direct relationship between the effects of the lipid composition on membrane affinity and membrane permeation.

In order to analyze these findings in more detail, we simulated the permeation kinetics based on the model depicted above. The solute concentration in the liposomal lumen was described as a function of time by a differential equation system with the rate constants for partitioning and translocation. We implemented a simulation algorithm to solve the system numerically and thus, to simulate and predict the process of permeation. With this model we were able to mimic our experimental findings and to delineate the controversial relationship between membrane affinity and membrane permeation.

\section{MATERIALS AND METHODS}

\section{Chemicals}

2-Hydroxynicotinic acid (2-OH-NA, \#55966), anthranilic acid (AA, \#10680), 3,5-dichlorobenzoic acid (DCBA, \#35321) and salicylic acid (SA, \#84210) were purchased from Fluka (Buchs, Switzerland). $\mathrm{TbCl}_{3}$ hexahydrate (\#21290-3) was supplied by Aldrich (Buchs, Switzerland). ${ }^{14} \mathrm{C}$-salicylic acid (\#NEC263) was purchased from PerkinElmer (Boston, MA), ${ }^{14}$ C-anthranilic acid (\#30455-7) from Sigma (Buchs, Switzerland) and ${ }^{14} \mathrm{C}$-2-hydroxynicotinic acid (\#1140) from American Radiolabeled Chemicals (St. Louis, MO). Egg phosphatidylcholine $(\mathrm{PhC})$ grade 1 and phosphatidylinositol (PhI) were supplied by Lipid Products (Nutfield, UK), cholesterol (Chol, \#C-8667) was from Sigma (Buchs, Switzerland) and steraylamine (StAm, \#74750) from Fluka (Buchs, Switzerland). All other chemicals used were of analytical grade.

\section{Potentiometric Determination of $\mathrm{pK}_{\mathrm{a}}$ and $\log \mathrm{P}_{\mathrm{Octanol}}$}

The $p K_{a}$ and $\log P_{\text {Octanol }}$ values of the studied ACAs were determined potentiometrically at $25^{\circ} \mathrm{C}$ with the PCA 101 instrument from Sirius Analytical Instruments (Forest Row, UK) as described elsewhere (14).

\section{Preparation of the Liposomes}

Liposomes were prepared by extrusion $(18,19)$. For permeation studies $\mathrm{Tb}^{3+}$-containing liposomes were prepared with $0.2 \mathrm{mmol} \mathrm{TbCl}_{3}$ hexahydrate and $100 \mathrm{mg}$ of the respective lipids ( $\mathrm{PhC}, \mathrm{PhC} / \mathrm{Chol}, \mathrm{PhC} / \mathrm{PhI}, \mathrm{PhC} / \mathrm{StAm})$ as described in Thomae et al. (14). Liposomes containing Chol and StAm were extruded through membranes with 800 and $400 \mathrm{~nm}$ pores prior to the extrusion through $200 \mathrm{~nm}$ pores (Nucleopore polycarbonate membranes, Corning, Acton, MA). External $\mathrm{Tb}^{3+}$ was separated from the liposomes on a Sephadex G-25 PD-10 desalting column (Amersham Biosciences, Freiburg, Germany) with $0.2 \mathrm{M} \mathrm{NaCl}$. The liposome fraction contained about 20 to $30 \mathrm{mg}$ lipids per $\mathrm{ml}$. The local concentration of $\mathrm{Tb}^{3+}$ in the liposomal lumen was between 5 and $7 \mathrm{mM}$ (19). $\mathrm{Tb}^{3+}$-containing liposomes were used within a few hours after preparation. Liposomes for partition studies were prepared without $\mathrm{Tb}^{3+}$ as described above, omitting the separation step at the end. The $\mathrm{Tb}^{3+}$-free liposomes were stored at $4^{\circ} \mathrm{C}$ and used within 3 days.

\section{Characterization of the Liposomes}

The average size and the size distribution of all liposome preparations were analyzed by dynamic light scattering with a Zetasizer 3000 HSA (Malvern Instruments, Malvern, UK). The $\mathrm{Tb}^{3+}$-containing liposomes showed an average mean hydrodynamic diameter between 140 and $180 \mathrm{~nm}$ and $\mathrm{Tb}^{3+}$ free liposomes between 120 and $200 \mathrm{~nm}$, depending on the lipid composition. The polydispersity indices of both types of liposomes were $<0.2$ corresponding to a size variance of maximal $45 \%$ assuming a monomodal distribution. $\mathrm{Tb}^{3+}$-free StAm-containing liposomes showed higher polydispersity indices $(<0.4$, corresponding to a size variance of $63 \%)$. All liposome preparations were further characterized by their zeta potential $\zeta$ at $\mathrm{pH} 6.8$ using the Zetasizer 3000 HSA. The zeta potential was $5 \pm 4 \mathrm{mV}$ for $\mathrm{PhC}$ and $\mathrm{PhC} / \mathrm{Chol}$ liposomes and $-5 \pm 4 \mathrm{mV}$ and $-23 \pm 4 \mathrm{mV}$ for $\mathrm{PhC} / \mathrm{PhI} 94 / 6(\mathrm{~mol} / \mathrm{mol})$ and $\mathrm{PhC} / \mathrm{PhI} 70 / 30$, respectively. For $\mathrm{Tb}^{3+}$-containing $\mathrm{PhCl}$ StAm $85 / 15$ liposomes $\zeta$ was $20 \pm 4 \mathrm{mV}$, while it was $40 \pm 4 \mathrm{mV}$ for $\mathrm{Tb}^{3+}$-free PhC/StAm 85/15 liposomes. Except for $\mathrm{PhCl}$ StAm liposomes, $\zeta$ of $\mathrm{Tb}^{3+}$-containing liposomes were similar to the values of the corresponding $\mathrm{Tb}^{3+}$-free liposomes, indicating that the binding or adsorption of $\mathrm{Tb}^{3+}$ to the membrane surface was negligible. The lipid composition of all liposome preparations were checked by thin layer 
chromatography (20). No significant deviations from the lipid composition of the starting lipid mixture were detected. At the end of the partitioning experiments the lipids were quantified by HPLC according to the method of Singh et al. (21).

\section{Permeation Assay}

The entry kinetics of the ACAs into the $\mathrm{Tb}^{3+}$-containing aqueous liposomal lumen were determined by luminescence measurements on a Synergy HT plate reader (Bio-Tek Instruments, Winooski, VT) as described recently (14). Permeant concentrations were between 5 and $50 \mu \mathrm{M}$ and lipid concentrations between 1 and $2 \mathrm{mg} / \mathrm{ml}$. The $\mathrm{pH}$ was adjusted with the universal buffer solution (SUBS, 22). The permeation kinetics were concentration-independent in the studied ACA concentration range (data not shown). The assays were run for $30-60 \mathrm{~min}$. The $\mathrm{pH}$ was stable within $0.1 \mathrm{pH}$ units during all kinetic measurements.

Permeation experiments at $\mathrm{pH} 2.5(\mathrm{HCl} / \mathrm{NaCl}$ buffer $)$ and 6.5 (MOPS buffer) with SA and liposomes with a buffered lumen, i.e. prepared in presence of buffer, revealed similar permeation coefficients (data not shown) as found for SA with the liposomes prepared in absence of buffer in this work. As discussed earlier, no $\mathrm{pH}$-gradient is expected under our experimental conditions (14).

\section{pH-dependent Partitioning in the Liposome System}

The affinities of ${ }^{14} \mathrm{C}-\mathrm{SA},{ }^{14} \mathrm{C}-\mathrm{AA}$ and ${ }^{14} \mathrm{C}-2-\mathrm{OH}-\mathrm{NA}$ to liposomal membranes were determined by a standardized equilibrium dialysis system (22). The $\mathrm{pH}$ was adjusted with SUBS as indicated, the concentration of the acids was $10^{-7} \mathrm{M}$. The concentration of lipids was $3 \mathrm{mg} / \mathrm{ml}$ for SA and AA $(\leq \mathrm{pH}$ 6), $30 \mathrm{mg} / \mathrm{ml}$ for SA and AA $(\mathrm{pH} 7)$ and $50 \mathrm{mg} / \mathrm{ml}$ for 2-OH-NA.

Distribution coefficients D were calculated according to Eq. 1 (14):

$$
D=\left(c_{\mathrm{LB}}-c_{\mathrm{B}}\right) \times V_{\mathrm{LB}} /\left(c_{\mathrm{B}} \times V_{\text {lipids }}\right)+1
$$

where $c_{\mathrm{LB}}$ is the molar solute concentration in the liposomecontaining chamber and $c_{\mathrm{B}}$ in the buffer chamber. The symbol $V_{\mathrm{LB}}$ denotes the sample volume of the liposome suspension, $V_{\text {lipids }}$ the volume of the lipophilic phase, i.e. the lipid bilayers. This volume was calculated from the lipid concentrations in the dialysis cells, which were determined by HPLC (see above).

\section{Data Analysis}

Data were analyzed with the solver tool of Microsoft Excel:mac and with SigmaPlot (Version 10, Systat Software Inc.). The luminescence/time curves of the permeation experiments $(I(t)=f(t))$ were fitted with a biexponential function according to Thomae at al. (14) (Eq. 2),

$$
\begin{aligned}
I(t)= & I(\max )_{I}+I(\max )_{I I}+I(0) \\
& -\left(I(\max )_{I} \times e^{-k_{I} t}+I(\max )_{I I} \times e^{-k_{I I} t}\right)
\end{aligned}
$$

where $I(\max )$ denotes the maximal luminescence of the respective exponential, $I(0)$ the luminescence at time 0 , i.e. the intersection with the ordinate, and $k$ the rate constant of the respective exponential. Indices in Eq. 2 refer to the fast $(I)$ and the slow (II) phases. Apparent permeation coefficients Perm $_{\text {app }}$ were calculated from $k_{I}$ and the liposomal radius $r$ as follows (Eq. 3):

$$
\operatorname{Perm}_{\mathrm{app}}=k_{I} \times r / 3
$$

where $r / 3$ is used as an approximation for the ratio between the total inner aqueous volume of the liposomes and the total membrane area. The radius $r$ was estimated from the hydrodynamic mean diameter of the liposomes (see above). Permeation coefficients were expressed as their logarithmic values $\log$ Perm $_{\mathrm{app}}$. Intrinsic permeation coefficients of the net neutral $\left(\log\right.$ Perm $\left.^{A H}\right)$, anionic $\left(\log\right.$ Perm $\left.^{A^{-}}\right)$and di-protonated $\left(\log\right.$ Perm $^{A H_{2}^{+}}$) compounds were fitted from the $\log$ Perm $_{\text {app }} / \mathrm{pH}$ profiles according to Eq. 4:

$\log$ Perm $_{\text {app }}=\log \left(\alpha^{A H_{2}^{+}} \times 10^{\log P e r m^{A H_{2}^{+}}}+\alpha^{A H} \times 10^{\log P e r m^{A H}}+\alpha^{A^{-}} \times 10^{\log P e r m^{A^{-}}}\right)$

where $\alpha^{A H_{2}^{+}}, \alpha^{A H}$ and $\alpha^{A^{-}}$are the molar fractions of the diprotonated, net neutral and deprotonated acids $(14,23)$.

The $\mathrm{pH}$-dependent distribution $D$ of the acids between the lipid bilayer and the aqueous phase was fitted as described elsewhere (6) according to Eq. 5:

$\log D=\log \left(\alpha^{A H_{2}^{+}} \times 10^{\log P^{A H_{2}^{+}}}+\alpha^{A H} \times 10^{\log P^{A H}}+\alpha^{A^{-}} \times 10^{\log P^{A^{-}}}\right)$

where $P^{A H_{2}^{+}}$is the partition coefficient of the di-protonated, $P^{A H}$ of the net neutral and $P^{A^{-}}$of the deprotonated compound.

The $p K_{a}$ values used to calculate $\alpha$ were determined by potentiometric titration (see above). For the analysis of the permeation and partitioning data revealed with liposomes containing charged lipids, the experimental $\mathrm{pH}$ values were corrected for the estimated difference $(\Delta \mathrm{pH})$ between the bulk $\mathrm{pH}(\mathrm{pH}($ bulk $))$ and the $\mathrm{pH}$ of the stationary buffer layer at the membrane ( $\mathrm{pH}($ at membrane) ) according to Krämer et al. (6), Eq. 6:

$$
\Delta \mathrm{pH}=\mathrm{pH}(\text { bulk })-\mathrm{pH}(\text { at membrane })=-\frac{F \times \zeta}{2.303 \times R T}
$$

where $F$ is the Faraday constant and $R$ the gas constant.

\section{Description of the Permeation Process by a Differential System of Equations and Numerical Solution Thereof}

Permeation kinetics were simulated based on the rate constants of the equilibria for partitioning and translocation as described in Eq. 7, which is in agreement with the model for membrane permeation by Lauger et al. (3).

$A_{a o} \underset{k_{-1}}{\stackrel{k_{1}}{\rightleftharpoons}} A_{l o} \underset{k_{-2}}{\stackrel{k_{2}}{\rightleftharpoons}} A_{l i} \underset{k_{-3}}{\stackrel{k_{3}}{\rightleftharpoons}} A_{a i} \underset{k_{-4}}{\stackrel{k_{4}}{\rightleftharpoons}} A_{T b}$ 
$A_{\text {ao }}$ denotes the solute $A$ in the outer aqueous phase, $A_{\text {lo }}$ and $A_{\mathrm{li}}$ the solute in the outer and inner lipid leaflet, respectively, and $A_{\text {ai }}$ stands for the solute in the liposomal lumen. The luminal solute ligated to entrapped $\mathrm{Tb}^{3+}$ in the liposomes is indicated by $A_{\mathrm{Tb}}$. The partitioning into the membrane is described by the rate constants $\mathrm{k}_{1}$ and $\mathrm{k}_{-3}, \mathrm{k}_{-1}$ and $\mathrm{k}_{3}$ are the dissociation rate constants. The ratios $\mathrm{k}_{1} / \mathrm{k}_{-1}$ and $\mathrm{k}_{-3} / \mathrm{k}_{3}$ equal the partition coefficient $P$. The translocation rate constants are defined as $\mathrm{k}_{2}$ and $\mathrm{k}_{-2}$. The rate constant $\mathrm{k}_{4}$ describes the 1:1 complex formation between free $A$ and $\mathrm{Tb}^{3+}$ and $\mathrm{k}_{-4}$ the dissociation of the complex.

The following conditions were considered for the membrane permeation model described in Eq. 7: (1) the complex formation $\left(\mathrm{k}_{4}\right)$ is faster than the overall permeation process as shown in Thomae et al. (14). Control experiments revealed that under our experimental conditions the complex formation had no influence on the overall permeation coefficients (data not shown). Thus, both $\mathrm{k}_{4}$ and $\mathrm{k}_{-4}$ were not included in the simulations. Simulations including the equilibrium for the complex formation revealed a range where $\mathrm{k}_{4}{ }^{\prime}$ and $\mathrm{k}_{-4}{ }^{\prime}$ were indeed without effect on the permeation coefficients (data not shown). (2) The bilayer is symmetrical and the inner and outer leaflets have equal volumes and thus (Eq. 8),

$$
k_{2}=k_{-2}
$$

(3) The solute concentrations in the aqueous and bilayer phases had to be normalized to a reference volume and thus the rate constants $\mathrm{k}$ in Eq. 7 were converted to the apparent rate constants k' for a normalized volume by the general Eq. 9:

$$
k^{\prime}=k \times \frac{V_{\text {norm }}}{V_{\mathrm{x}}}
$$

with $V_{\text {norm }}$ as the reference volume for normalization and $V_{\mathrm{x}}$ the volume of the respective origin phase in the experimental setup.

With these considerations the rate laws for Eq. 7 can be written as:

$$
\begin{gathered}
\frac{\mathrm{d}\left[A_{\mathrm{ao}}\right]}{\mathrm{d} t}=-k_{1}^{\prime} \times\left[A_{\mathrm{ao}}\right]+k_{-1}^{\prime} \times\left[A_{\mathrm{lo}}\right] \\
\frac{d\left[A_{l o}\right]}{d t}=k_{1}^{\prime} \times\left[A_{a o}\right]-\left(k_{-1}^{\prime}+k_{2}{ }^{\prime}\right) \times\left[A_{l o}\right]+k_{-2}^{\prime} \times\left[A_{l i}\right] \\
\frac{\mathrm{d}\left[A_{\mathrm{li}}\right]}{\mathrm{d} t}=k_{2}^{\prime} \times\left[A_{\mathrm{lo}}\right]-\left(k_{-2}{ }^{\prime}+k_{3}{ }^{\prime}\right) \times\left[A_{\mathrm{li}}\right]+k_{-3}{ }^{\prime} \times\left[A_{\mathrm{ai}}\right] \\
\frac{\mathrm{d}\left[A_{\mathrm{ai}}\right]}{\mathrm{d} t}=-k_{-3}{ }^{\prime} \times\left[A_{\mathrm{ai}}\right]+k_{3}{ }^{\prime} \times\left[A_{\mathrm{li}}\right]
\end{gathered}
$$

We implemented a simulation algorithm to solve numerically the differential equation system Eqs. 10-13 with different configurations of the rate constants. The numerical solution of the differential equations revealed triexponential equations of the form (Eq. 14),

$$
\left[A_{\mathrm{x}}\right](t)=a \times e^{-q t}+b \times e^{-r t}+c \times e^{-s t}+d
$$

where $A_{x}(t)$ represents $A$ in phase x at time $t$ and $q, r, s$ are composed of the single rate constants $\mathrm{k}_{1}{ }^{\prime}$ to $\mathrm{k}_{-3}{ }^{\prime}$.
To compare the overall permeation rates, i.e. $\mathrm{d}\left[A_{\mathrm{ai}}\right] / \mathrm{d} t$, we introduced $t_{90}$, the time when $90 \%$ of the plateau value $\left[A_{\mathrm{ai}}\right](\mathrm{t} \rightarrow \infty)$ is reached. Note that an increase in $t_{90}$ reflects a decrease in the permeation rate. The value of $t_{90}$ was in good agreement with the time point when $50 \%$ of the final concentrations were reached. The simulation algorithm was implemented in ANSI-C (24) and Java. Both source codes are available under http://www.biopharmacy.ethz.ch/simulations/ PermSim/index.html.

\section{Estimation of the Volumes of the Aqueous and Lipid Phases}

Assuming that liposomes are spheres, the total volume of one average liposome $V_{\text {liposome }}$ was calculated according to Eq. 15

$$
V_{\text {liposome }}=\frac{1}{6} \pi \times d^{3}
$$

with $d$ corresponding to the hydrodynamic diameter obtained by dynamic laser light scattering (see above). The volume of the inner aqueous phase $V_{\text {ai }}$ and the volume of the lipid bilayer $V_{\text {bilayer }}$ of one average liposome was calculated from $d$ and the bilayer thickness $h(h \sim 4 \mathrm{~nm}(25))$ according to Eqs. 16 and 17, respectively:

$$
\begin{aligned}
V_{\text {inner }} & =\frac{1}{6} \pi \times(d-2 h)^{3} \\
V_{\text {bilayer }} & =V_{\text {liposome }}-V_{\text {inner }}
\end{aligned}
$$

With the amount of lipids [mg] used for the assay the total lipid volume $V_{\text {lipids }}$ was approximated with a lipid density $\rho=1.0 \mathrm{~g} \cdot \mathrm{ml}^{-1}(25)$. The number of liposomes $N_{\text {liposomes }}$ resulted from Eq. 18 .

$$
N_{\text {liposomes }}=\frac{V_{\text {lipids }}}{V_{\text {bilayer }}}
$$

The total volume of inner aqueous $V_{\text {ai }}$ phase is related to $N_{\text {liposomes }}$ by Eq. 19:

$$
V_{\mathrm{ai}}=N_{\text {liposome }} \times V_{\text {inner }}
$$

The volume of the outer aqueous phase $V_{\text {ao }}$ is calculated from the total sample volume ( $\left.V_{\text {total }}\right)$ as shown in Eq. 20.

$$
V_{\text {ao }}=V_{\text {total }}-\left(N_{\text {liposome }} \times V_{\text {liposome }}\right)
$$

The total volume of all outer $\left(V_{\text {lo }}\right)$ or inner $\left(V_{\text {li }}\right)$ lipid leaflets, respectively, was estimated by Eq. 21:

$$
V_{\mathrm{li}} \approx V_{\mathrm{lo}}=\frac{V_{\text {lipids }}}{2}
$$

With the standard conditions of the permeation assay, i.e. $d \sim 200 \mathrm{~nm}$ and $c_{\text {lipid }} \sim 2 \mathrm{mg} / \mathrm{ml}$, Eqs. 15-21 reveal the following volume ratios (Eq. 22).

$$
V_{\mathrm{ao}}: V_{\mathrm{lo}}: V_{\mathrm{li}}: V_{\mathrm{ai}}=98.6: 0.1: 0.1: 1.2 \approx 1000: 1: 1: 10
$$

\section{Relationship between True and Apparent Rate Constants}

As the volumes of the aqueous and lipid phases are not equal (Eq. 22) in the permeation assay, the true rate con- 
stants $\mathrm{k}$ have to be transformed into apparent rate constants k'. Considering the partition coefficient $P$ and applying the volume ratios described above the ratios of $\mathrm{k}_{1}{ }^{\prime} / \mathrm{k}_{-1}{ }^{\prime}$ and $\mathrm{k}_{3}{ }^{\prime}$ / $\mathrm{k}_{-3}{ }^{\prime}$ can be calculated with Eqs. 23-25.

$$
\begin{gathered}
P=\frac{k_{1}}{k_{-1}}=\frac{k_{-3}}{k_{3}} \\
\frac{k_{1}^{\prime}}{k_{-1}^{\prime}}=P \times \frac{V_{\mathrm{lo}}}{V_{\mathrm{ao}}}=P \times \frac{1}{1000} \\
\frac{k_{-3}^{\prime}}{k_{3}{ }^{\prime}}=P \times \frac{V_{\mathrm{li}}}{V_{\mathrm{ai}}}=P \times \frac{1}{10}=100 \times \frac{k_{1}^{\prime}}{k_{-1}^{\prime}}
\end{gathered}
$$

Normalization to $V_{\text {norm }}$ according to Eq. 9 revealed the apparent rate constants (Eqs. 26-29) for a symmetrical bilayer with equal conditions in the outer and inner aqueous phases.

\section{RESULTS}

\section{Physicochemical Characteristics of the Test Compounds}

Three aromatic carboxylic acids SA, AA and 2-OH-NA of similar molecular size but with considerable differences in their acidity/basicity behavior and octanol/water partition coefficients were chosen as test compounds for the comparison of membrane affinity and permeation. A fourth compound, DCBA, was tested in the permeation assay with $\mathrm{PhC}$ liposomes to shed light on the influence of intramolecular hydrogen bonding on the permeation/pH profile.

The structural similarity of the test compounds allowed to focus on the influence of their ionization state, membrane affinity and hydrogen bonding capacity on lipid bilayer permeation in different liposome systems. Table I shows the $p K_{a}$ values of the compounds and the $\log P_{\text {Octanol }}$ values of their ionization species as determined by potentiometric titration. SA and DCBA are monoprotic acids. AA is an amphoteric molecule with two ionizable groups; the net neutral species is predominating in the $\mathrm{pH}$ range between the two $p K_{a}$ values. The titration of $2-\mathrm{OH}-$ NA between $\mathrm{pH} 2$ and 10 revealed one ionizable group within this $\mathrm{pH}$ range. The $\log P_{\text {Octanol }}$ of the net neutral compounds were 2.48 for SA, 1.20 for AA and -0.13 for $2-\mathrm{OH}-\mathrm{NA}$.

\section{Affinity- and Permeation/pH Profiles of the Test Compounds in the Egg PhC Liposome System}

The membrane affinities of SA, AA and 2-OH-NA, expressed as $\log D$, were determined by equilibrium dialysis between $\mathrm{pH} 1.5$ and 7.5. The $\log D$ values were $\mathrm{pH}$-dependent, following Henderson-Hasselbalch functions. The experimental data are shown in Fig. 1a together with the fitted distribution profiles according to (14) and Eq. 5. The fitted $\log P$ values of

\begin{tabular}{|c|c|c|c|c|}
\hline & SA & $\mathbf{A A}$ & 2-OH-NA & DCBA \\
\hline Structure & & & & \\
\hline$p K_{a}^{A H_{2}{ }^{+} / A H a}$ & - & $2.15 \pm 0.11$ & $<2^{d}$ & - \\
\hline$p K_{a}^{A H / \Lambda^{-} a}$ & $2.75 \pm 0.03$ & $4.76 \pm 0.01$ & $4.97 \pm 0.01$ & $3.18 \pm 0.02$ \\
\hline $\log \mathrm{P}_{2}^{\mathrm{AH}}{ }_{2}^{+}$Octanol $b$ & - & n.d. ${ }^{d}$ & n.d. ${ }^{d}$ & - \\
\hline $\log P^{A H}{ }_{\text {Octanol }}^{b}$ & $2.48 \pm 0.01$ & $1.20 \pm 0.02$ & $-0.13 \pm 0.05$ & $3.61 \pm 0.08$ \\
\hline $\log P^{A^{-}}$Octanol $^{b}$ & $<-1.7^{c}$ & $<-1.7^{c}$ & $-1.05 \pm 0.11$ & $-0.4 \pm 0.3$ \\
\hline
\end{tabular}
the different ionization species are shown in Table II.

Table I. Structures, $p K_{a}$ and $\log P_{\text {Octanol }}$ of the Studied ACAs

\footnotetext{
${ }^{a}$ Mean value \pm standard deviation; $n=3 ; T 25^{\circ} \mathrm{C}$.

${ }^{b}$ Fitted value \pm estimated standard error; $T 25^{\circ} \mathrm{C}$.

${ }^{c}$ Value below detection limit.

${ }^{d}$ Outside electrode calibration range.
} 
SA
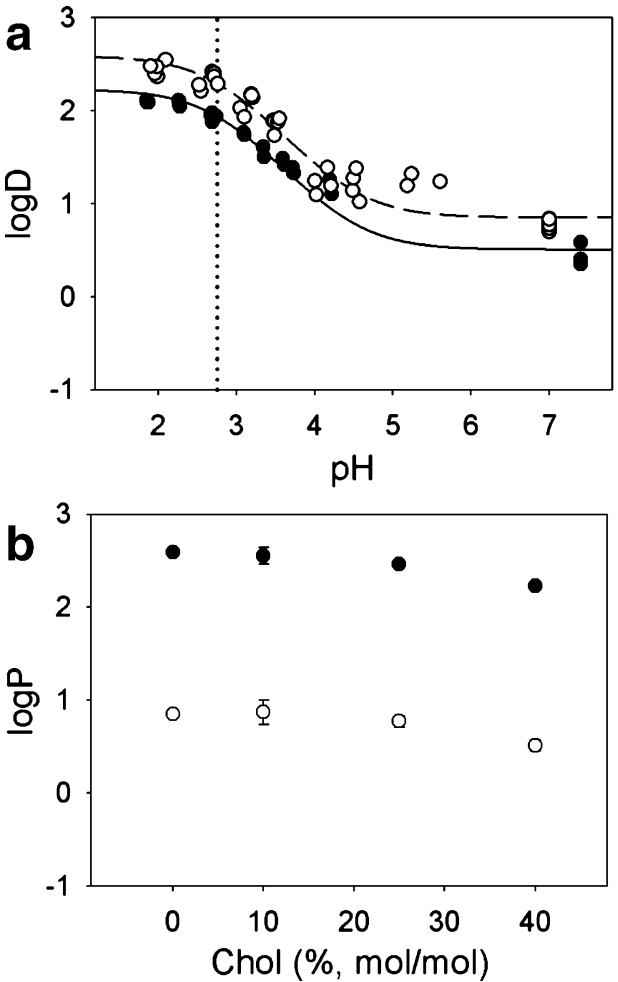

AA
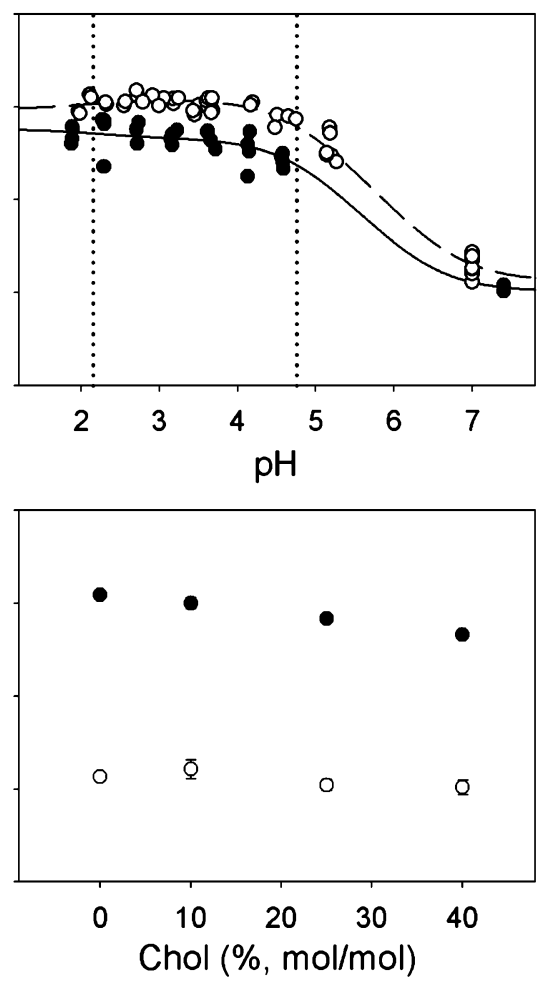

2-OH-NA

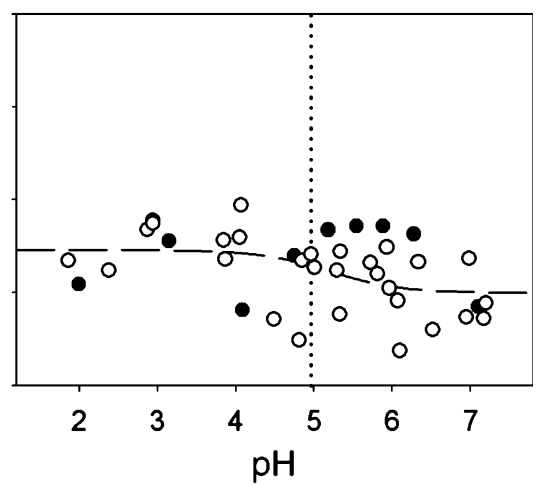

Fig. 1. Affinity to $\mathrm{PhC}$ and $\mathrm{PhC} / \mathrm{Chol}$ bilayers: a Membrane affinity of $\mathrm{SA}, \mathrm{AA}$ and 2-OH-NA to $\mathrm{PhC}(\mathrm{O})$ and $\mathrm{PhC} / \mathrm{Chol} 60 / 40(\bullet)$ liposomes. $\log D / \mathrm{pH}$ profiles were fitted based on the Henderson-Hasselbalch equation with the titrated $p K_{a}$ of the respective acid (dotted lines) and considering the partitioning of all ionization species ( $\mathrm{PhC}$, broken line; $\mathrm{PhC} / \mathrm{Chol} 60 / 40$, solid line). b Fitted $\log P$ values (error bars, estimated standard errors) of the different ionization species $\left(\log P^{A H} \bullet ; \log P^{A^{-}} \mathrm{O}\right)$ for SA and AA in PhC and all tested PhC/Chol liposomes. Data are from two or three independent experiments.

Comparing the net neutral species $(\mathrm{AH})$, SA had the highest PhC membrane affinity, $\log P_{P h C}^{A H}$ was 2.59 , followed by AA with 2.08. The membrane affinity $(\log D)$ of 2-OH-NA was in general $<1$, leading to scattering of the data, hence $\log P$ values were not fitted in any of the systems. This sequence is the same as observed for the $\log P$ values of the net neutral species in the octanol/water partition system (see Table I). The membrane affinities of the anionic species $\left(A^{-}\right)$ of SA and AA were about 55 and 90 times lower than those of the corresponding neutral species $\left(\log P_{P h C}^{A^{-}} \quad 0.85\right.$ and 0.13 , respectively). The affinity of the di-protonated species $\mathrm{AH}_{2}^{+}$of AA was similar to the affinity of the net neutral species.

The permeation across $\mathrm{PhC}$ bilayers was measured in a $\mathrm{pH}$ range between 2 and 7. Fig. 2a shows the experimental logarithmic permeation coefficients $\left(\log\right.$ Perm $\left._{\text {app }}\right)$ of SA, AA and 2-OH-NA together with the fitted permeation profiles according to (14) and Eq. 4. As described recently for SA and 2-OH-NA (14), the permeation profile of AA deviated significantly from the $\mathrm{pH}$-partition hypothesis. The permeation of the anionic species controlled the permeation around the physiological $\mathrm{pH}$ 7. This is indicated by the plateau of the $\log$ Perm $_{\text {app }} / \mathrm{pH}$ profile in this $\mathrm{pH}$ range. The $\log$ Perm values are shown in Table III. The cationic species of AA permeated faster than the corresponding net neutral and anionic species as calculated from the experimental data according to Eq. 4. No direct relationship can be observed between the membrane affinity and the membrane permeation of the tested compounds in PhC liposomes. Neither did the $\log P_{\text {Octanol }}$ correlate with bilayer permeation.

Table II. Membrane Affinity Parameters

\begin{tabular}{clcccc}
\hline & & PhC & PhC/Chol 60/40 & PhC/PhI 70/30 & PhC/StAm 85/15 \\
\hline SA & $\log P^{A H}$ & $2.59 \pm 0.03$ & $2.23 \pm 0.02$ & $2.41 \pm 0.04$ & $2.59 \pm 0.04$ \\
& $\log P^{A^{-}}$ & $0.85 \pm 0.04$ & $0.51 \pm 0.06$ & $0.00 \pm 0.07$ & $1.60 \pm 0.02$ \\
AA & $\log P^{A H_{2}^{+}}$ & $1.97 \pm 0.08$ & n.d. $^{a}$ & n.d. $^{a}$ & $1.74 \pm 0.06$ \\
& $\log P^{A H}$ & $2.08 \pm 0.02$ & $1.66 \pm 0.03$ & $-0.40 \pm 0.32$ & $1.83 \pm 0.03$ \\
& $\log P^{A^{-}}$ & $0.13 \pm 0.04$ & $0.02 \pm 0.08$ & & $1.06 \pm 0.04$ \\
\hline
\end{tabular}

Fitted values \pm estimated standard errors are shown. The membrane affinities of all ionization species of 2-OH-NA were too low to be fitted reliably. ${ }^{a}$ n.d., not determined. 
SA
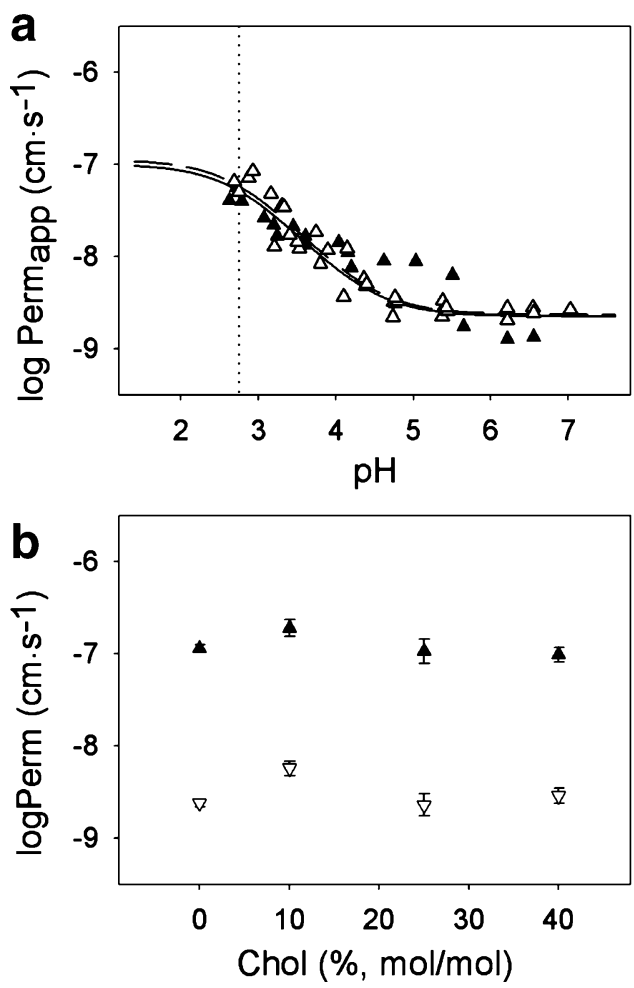

AA
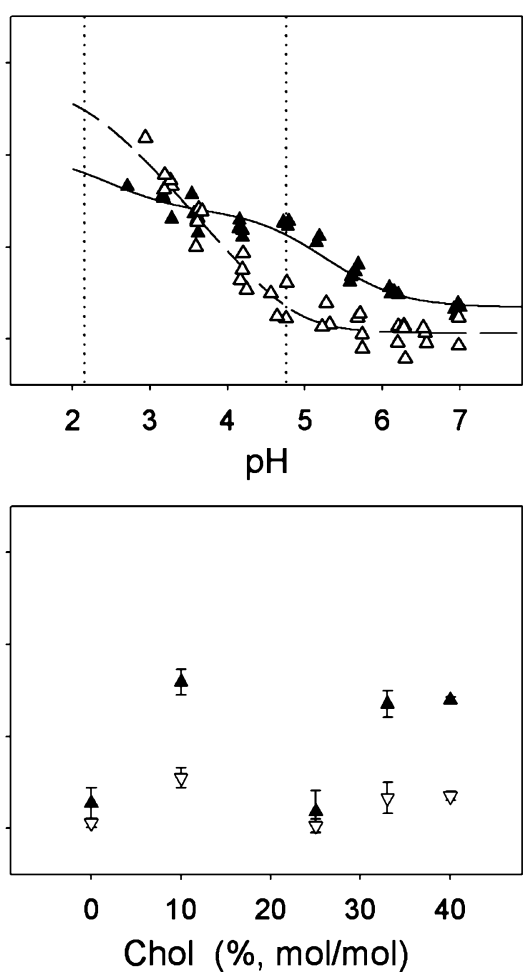

2-OH-NA
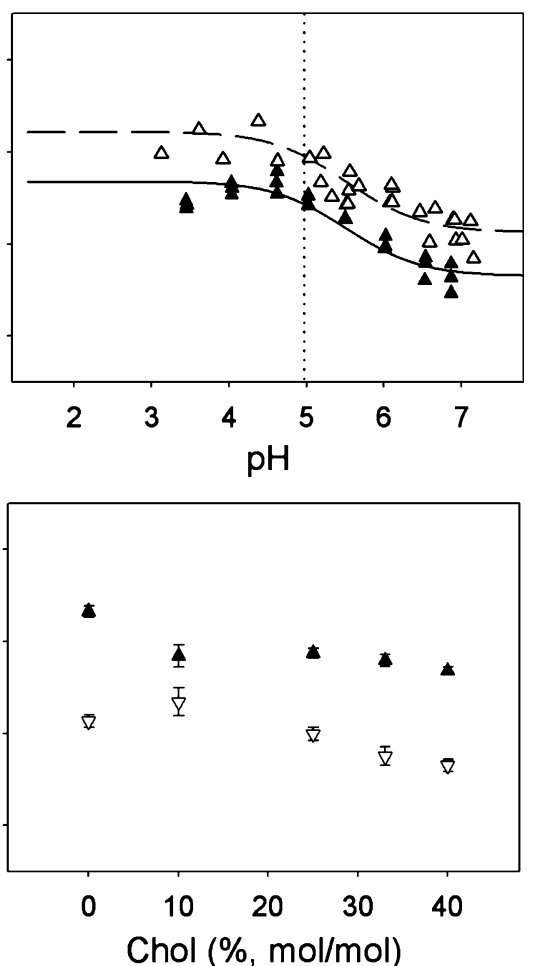

Fig. 2. Permeation across $\mathrm{PhC}$ and $\mathrm{PhC} / \mathrm{Chol}$ bilayers: a Membrane permeation of SA, AA and 2-OH-NA across $\mathrm{PhC}(\triangle)$ and $\mathrm{PhC} / \mathrm{Chol}(60 /$ 40) ( $\mathbf{\Delta})$ bilayers. The $\log$ Perm/pH profiles were fitted as described in Fig. 1 considering the permeation of all ionization species. b Fitted $\log$ Perm values for the different ionization species $\left(\log \mathrm{Perm}^{A H} \boldsymbol{\Lambda} ; \log P e r m^{A^{-}} \nabla\right)$ in $\mathrm{PhC}$ and all tested PhC/Chol liposomes. Data are from three independent experiments.

\section{Influence of the Intramolecular Hydrogen Bonding Capability on Bilayer Permeation}

The relatively high permeation rates of the anionic species of SA, AA and 2-OH-NA could be due to their capacity to form intramolecular hydrogen bonds and thus to delocalize their charge. We therefore investigated the $\mathrm{pH}$ dependent permeation of DCBA, which is unable to form such strong intramolecular hydrogen bonds, in $\mathrm{PhC}$ liposomes. As shown in Fig. 3 and Table III, permeation of DCBA at $\mathrm{pH} 7$ was anion-controlled, even in the absence of intramolecular hydrogen bonding possibilities.

\section{Influence of Chol on the Membrane Affinity of SA, AA and 2-OH-NA}

To investigate the influence of Chol on the membrane affinity of the three ACAs, equilibrium dialysis experiments were performed with either $\mathrm{PhC} / \mathrm{Chol}$ 90/10, $\mathrm{PhC/Chol} \mathrm{75/25}$ or $\mathrm{PhC} / \mathrm{Chol} 60 / 40$ liposomes. The comparison of the distribution profiles obtained with $\mathrm{PhC} / \mathrm{Chol}$ 60/40 liposomes and $\mathrm{PhC}$ liposomes is shown in Fig. 1a (see Supplementary Material Fig. S1 for all distribution profiles). The fitted $\log P$ values at all tested $\mathrm{PhC} / \mathrm{Chol}$ ratios are shown in Fig. 1b. The $\log P$ values of all ionization species at $40 \%(\mathrm{~mol} / \mathrm{mol})$ Chol

Table III. Membrane Permeation Parameters

\begin{tabular}{llcccc}
\hline & & PhC & PhC/Chol 60/40 & PhC/PhI 70/30 & PhC/StAm 85/15 \\
\hline SA & $\log$ Perm $^{A H}$ & $-6.94 \pm 0.04$ & $-7.01 \pm 0.08$ & $-7.01 \pm 0.12$ & $-7.10 \pm 0.30$ \\
& $\log$ Perm $^{A^{-}}$ & $-8.62 \pm 0.05$ & $-8.54 \pm 0.08$ & $-7.05 \pm 0.04$ & $-8.26 \pm 0.09$ \\
AA & $\log$ Perm $^{A H_{2}^{+}}$ & $-6.22 \pm 0.05$ & $-6.99 \pm 0.17$ & $-7.72 \pm 1.63$ & $a$ \\
& $\log$ Perm $^{A H}$ & $-8.73 \pm 0.17$ & $-7.61 \pm 0.04$ & $-7.03 \pm 0.08$ & $\geq-7.4$ \\
& $\log$ Perm $^{A^{-}}$ & $-8.94 \pm 0.05$ & $-8.65 \pm 0.05$ & $-7.91 \pm 0.14$ & $-8.63 \pm 0.14$ \\
2-OH-NA & $\log$ Perm $^{A H}$ & $-6.78 \pm 0.06$ & $-7.32 \pm 0.04$ & $-7.41 \pm 0.10$ & $-7.33 \pm 0.11$ \\
& $\log$ Perm $^{A^{-}}$ & $-7.87 \pm 0.07$ & $-8.35 \pm 0.07$ & $-7.59 \pm 0.10$ & $-7.68 \pm 0.07$ \\
DCBA & $\log$ Perm $^{A H}$ & $-7.17 \pm 0.06$ & n.d. $^{b}$ & n.d. $^{b}$ & n.d. ${ }^{b}$ \\
& $\log$ Perm $^{A^{-}}$ & $-8.45 \pm 0.05$ & n.d. $^{b}$ & n.d. $^{b}$ & n.d. \\
\end{tabular}

Fitted values $\left(\mathrm{cm} \cdot \mathrm{s}^{-1}\right) \pm$ estimated standard errors are shown.

${ }^{a}$ no luminescence signal

${ }^{b}$ n.d., not determined 


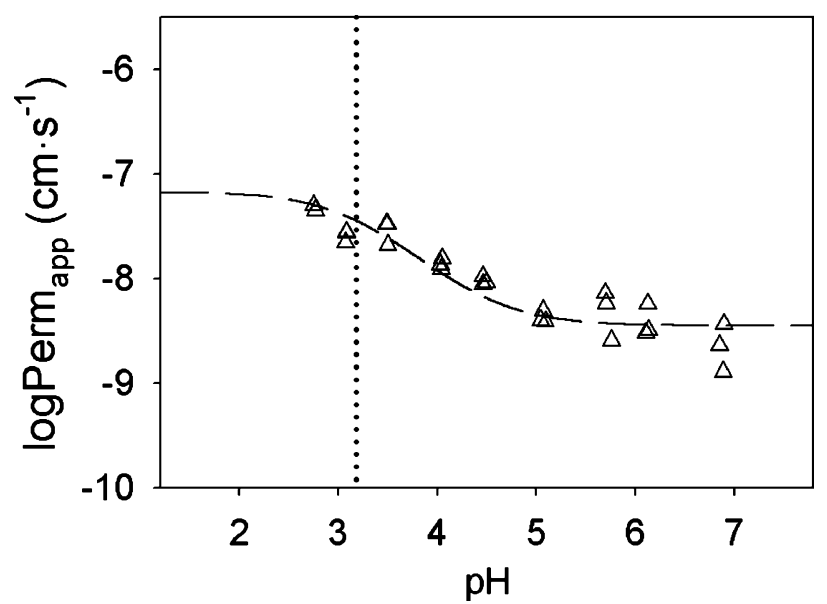

Fig. 3. Permeation/pH profile of DCBA in $\mathrm{PhC}$ liposomes. Fit as described in Fig. 2.

are given in Table II. For SA and AA, Chol reduced the membrane affinity at membrane contents $\geq 25 \%$. The membrane affinities of the cationic species $\mathrm{AH}_{2}^{+}$of AA are not well defined and therefore not shown.

\section{Influence of Chol on the Membrane Permeation}

To investigate the influence of Chol on the lipid bilayer permeation, $\log$ Perm $_{\text {app }}$ were determined with liposomes containing various amounts of Chol (see above) and compared to the results obtained with $\mathrm{PhC}$ liposomes. The influence of Chol on the $\log$ Perm $_{\mathrm{app}} / \mathrm{pH}$ profiles of the test compounds was not consistent with its effect on the $\log D / \mathrm{pH}$ profiles. The permeation profiles with the highest tested Chol amount (40\%) and the fitted $\log$ Perm values are shown in Fig. $2 \mathrm{a}$ and Table III, respectively. The permeation profiles of all tested $\mathrm{PhC/Chol} \mathrm{liposomes} \mathrm{are} \mathrm{shown} \mathrm{in} \mathrm{Fig.} \mathrm{S2} \mathrm{of} \mathrm{the} \mathrm{Supplementary}$ Material. Fig. 2b shows the fitted $\log$ Perm values of the single ionization species at all tested amounts of Chol.

The permeation of the neutral and anionic SA was not affected by 25 or $40 \%$ Chol in the bilayer. However, a significant increase of the permeation coefficient by a factor of 2 ( 0.3 in log units) was observed for both the neutral and charged species with $\mathrm{PhC/Chol} \mathrm{90/10} \mathrm{liposomes.}$

The net neutral species of AA permeated about 20 times faster across Chol-containing bilayers (except at $25 \% \mathrm{Chol}$ ) than across PhC bilayers, and Perm $^{A^{-}}$was increased by a factor of 3 . This major increase in $\log P e r m^{A H}$ led to a change in the shape of the permeation profile. Interestingly, $25 \% \mathrm{Chol}$ had no influence on the permeation profile of AA. The $\log \mathrm{Perm}^{A H_{2}^{+}}$ values were not well defined by the experimental data and were hence not fitted. The permeation of the net neutral and anionic 2-OH-NA was reduced by $\geq 25 \%$ Chol.

\section{Influence of the Negatively Charged PhI on the Membrane Affinity}

To explore the influence of the negatively charged $\mathrm{PhI}$ on the membrane affinity, equilibrium dialysis experiments
SA
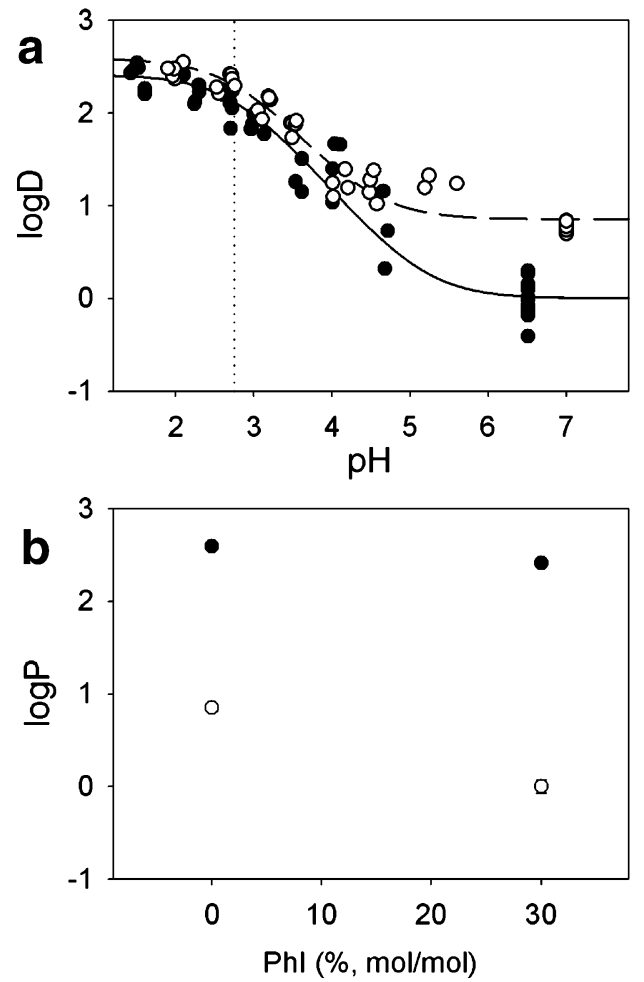

AA
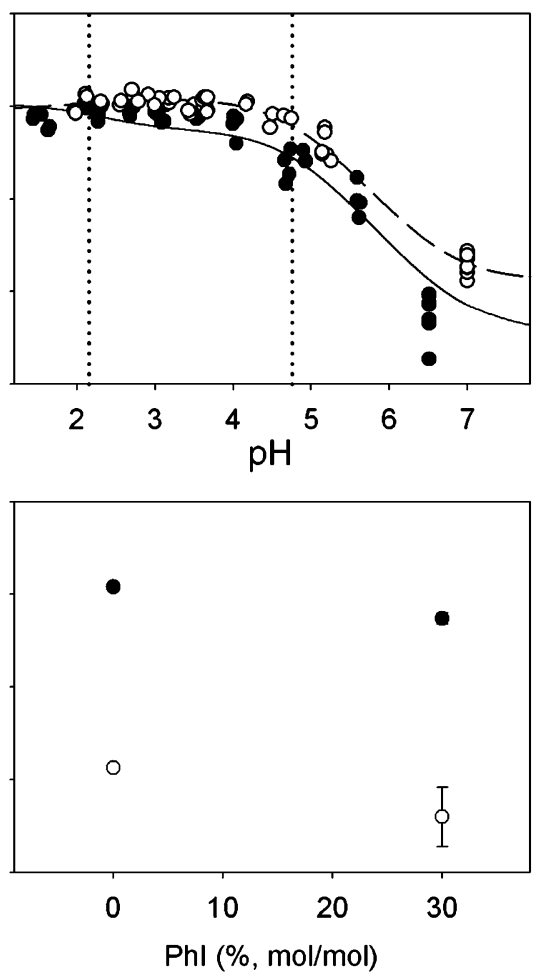

2-OH-NA

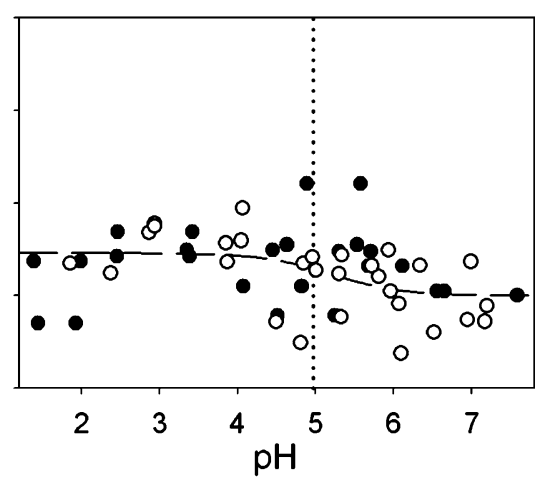

Fig. 4. Affinity to $\mathrm{PhC}$ and $\mathrm{PhC} / \mathrm{PhI}$ bilayers: (a) Membrane affinity of SA, AA and 2-OH-NA to $\mathrm{PhC}(\mathrm{O})$ and $\mathrm{PhC} / \mathrm{PhI} 70 / 30$ (•) liposomes. $\log D / \mathrm{pH}$ profiles were fitted as described in Fig. 1 ( $\mathrm{PhC}$, broken line; $\mathrm{PhC} / \mathrm{PhI} 70 / 30$, solid line). The $\mathrm{pH}$ values were corrected for the estimated $\mathrm{pH}$ difference between the bulk buffer (measured $\mathrm{pH}$ ) and the $\mathrm{pH}$ at the membrane surface. (b) Fitted $\log P$ values of the different ionization species $\left(\log P^{A H} \bullet ; \log P^{A^{-}} \mathrm{O}\right)$ for $\mathrm{SA}$ and $\mathrm{AA}$ in $\mathrm{PhC}$ and $\mathrm{PhC} / \mathrm{PhI} 70 / 30$ liposomes. Data are from two or three independent experiments. 
were performed with $\mathrm{PhC} / \mathrm{PhI} 70 / 30$ liposomes. Fig. 4 and Table II show the distribution profiles and the fitted $\log P$ values. PhI led to a significant reduction of the membrane affinities of the anionic species of SA and AA as compared to the egg $\mathrm{PhC}$ liposomes.

\section{Influence of PhI on the Permeation of the Test Compounds}

The influence of PhI on the membrane permeation was tested with $\mathrm{PhC} / \mathrm{PhI}$ 94/6 and $\mathrm{PhC} / \mathrm{PhI} 70 / 30$ liposomes. The permeation profiles and fitted $\log$ Perm values are shown in Fig. 5 and Table III and in the Supplementary material Fig. S3. As found for Chol, the effects of $\mathrm{PhI}$ on the permeation/pH profiles were not consistent with its effects on the distribution/ $\mathrm{pH}$ profiles.

The $\log P e r m^{A H}$ of SA did not change significantly in the presence of $\mathrm{PhI}$. While the permeation coefficient of the SA anion was not affected by $6 \% \mathrm{PhI}$ it was 38 -fold increased with $30 \% \mathrm{PhI}$. At this $\mathrm{PhC} / \mathrm{PhI}$ ratio no significant difference occurred between $\log$ Perm $^{A H}$ and $\log$ Perm $^{A^{-}}$, i.e. the neutral and the anionic species permeated with similar rate constants.

In the case of $\mathrm{AA}, 6 \% \mathrm{PhI}$ led to an increase of $\mathrm{Perm}^{\mathrm{AH}}$. A further increase of $\mathrm{Perm}^{A H}$ in $\mathrm{PhC} / \mathrm{PhI} 70 / 30$ liposomes affected the shape of the permeation profile in a similar manner as described for 10 and $>25 \%$ Chol. For 2-OH-NA, 6 and $30 \%$ of $\mathrm{PhI}$ led to a decrease of $\mathrm{Perm}^{A H}$.
Influence of the Positively Charged StAm on the Membrane Affinity

The influence of the positively charged lipid StAm on the membrane affinity was investigated using $\mathrm{PhC} / \mathrm{StAm}$ $85 / 15$ liposomes. The distribution profiles and fitted $\log P$ values are shown in Fig. 6 and Table II.

The partition coefficients of the SA and AA anions were significantly increased in presence of $15 \%$ of the positively charged StAm as compared to $\mathrm{PhC}$ bilayers. The membrane affinity of the net neutral species was decreased by a factor 2. The membrane affinity of the positively charged species of AA was not well defined but was clearly lower than in the $\mathrm{PhC}$ liposome system. Note that data were best fitted allowing a deviation of the inflection points of the distribution profiles of SA and AA (inflection point around $\mathrm{pH}$ 6) from the titrated $p K_{a}$ by +0.8 and $+1.4 \mathrm{pH}$ units, respectively.

\section{Influence of StAm on the Membrane Permeation}

Interestingly, the positively charged StAm had similar effects on the permeation of the anions of the three test compounds as the negatively charged PhI, although they had opposing effects on the membrane affinity (Fig. 7 and Table III). The permeation coefficients of the anionic SA and AA were enhanced by a factor of 2 with $15 \%$ StAm in PhC/StAm
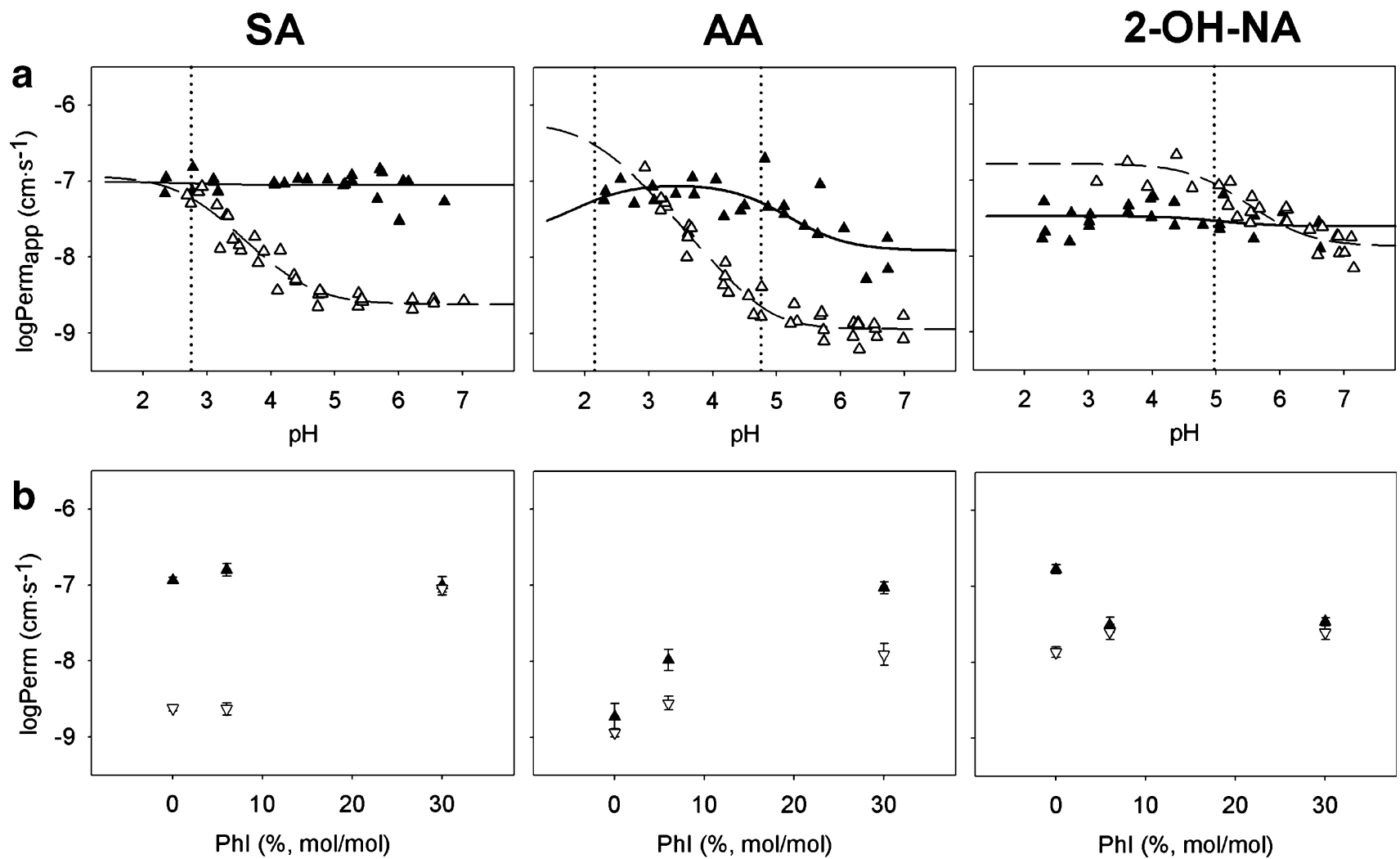

Fig. 5. Permeation across $\mathrm{PhC}$ and $\mathrm{PhC} / \mathrm{PhI}$ bilayers: a Membrane permeation of $\mathrm{SA}, \mathrm{AA}$ and 2-OH-NA across $\mathrm{PhC}(\triangle)$ and $\mathrm{PhC} / \mathrm{PhI}(70 / 30)$ $(\boldsymbol{\Delta})$ bilayers. The $\log P$ erm $/ \mathrm{pH}$ profiles were fitted as described in Fig. 1 considering the permeation of all ionization species and the $\mathrm{pH}$ difference between bulk buffer and membrane surface (see Fig. 4). b Fitted $\log$ Perm values for the different ionization species $\left(\log P e r m{ }^{A H} \boldsymbol{\Lambda}\right.$; $\log$ erm $\left.^{A^{-}} \nabla\right)$ in $\mathrm{PhC}$ and all tested $\mathrm{PhC} / \mathrm{PhI}$ liposomes. Data are from three independent experiments. 

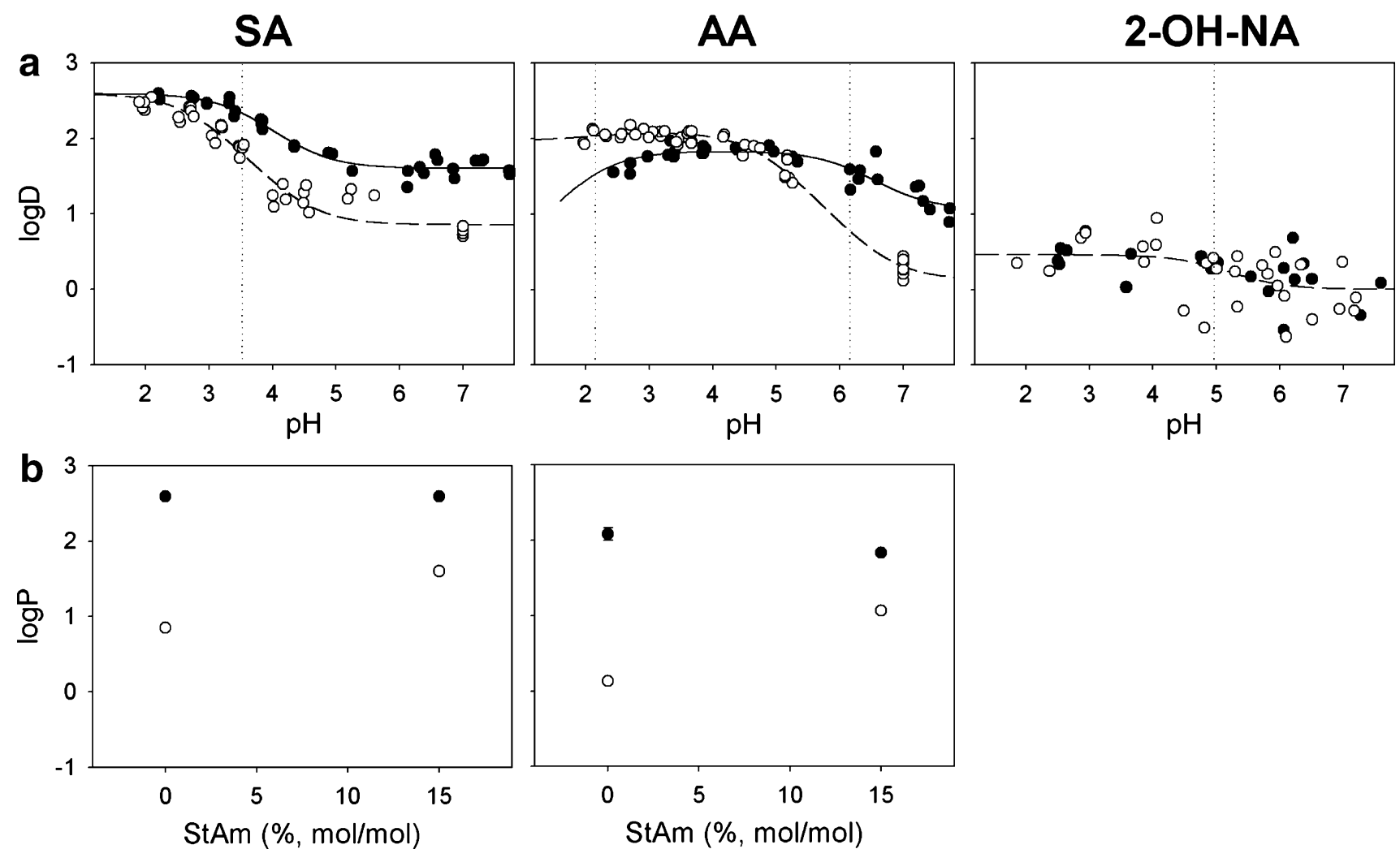

Fig. 6. Affinity to $\mathrm{PhC}$ and $\mathrm{PhC} / \mathrm{StAm}$ bilayers: a Membrane affinity of SA, AA and 2-OH-NA to $\mathrm{PhC}(\mathrm{O})$ and $\mathrm{PhC} / \mathrm{StAm} 85 / 15(\bullet)$ liposomes. $\log D / \mathrm{pH}$ profiles were fitted as described in Fig. 1 ( $\mathrm{PhC}$, broken line; $\mathrm{PhC} / \mathrm{Chol} 60 / 40$, solid line) allowing a deviation of the inflection points from the titrated $p K_{a}$ (pH values were corrected as described in Fig. 4). b Fitted $\log P$ values of the different ionization species $\left(\log P^{A H} \bullet ; \log P^{A^{-}} \mathrm{O}\right)$ for SA and $\mathrm{AA}$ in $\mathrm{PhC}$ and $\mathrm{PhC} / \mathrm{StAm} 85 / 15$ liposomes. Data are from two or three independent experiments.

liposomes as compared to $\mathrm{PhC}$ liposomes. No significant effect was seen on the permeation of the anionic 2-OH-NA. Below $\mathrm{pH} 4$ no AA luminescence was detectable in this system. The Perm $^{A H}$ of AA was therefore fitted with the data $>\mathrm{pH} 4$ (see Fig. 7a); the estimated $P e r m^{A H}$ was $>20$ times higher than in $\mathrm{PhC}$ liposomes (Table III).

\section{Simulations of Lipid Bilayer Permeation}

The permeation process across a lipid bilayer was simulated according to Eq. 7 taking into account the partitioning and translocation rate constants which were adjusted to our experimental conditions. The model applies for polar lipophilic permeants, which preferentially reside outside the middle plane of the lipid bilayer. Most drug compounds may therefore follow this model. It is easily adaptable for apolar compounds such as amiodarone which prefer the core region of the membrane (26) and may rather translocate by diffusion than by a flip flop event. In this case, diffusion is included in the partitioning rate constants and can be omitted in the simulation.

The ratios of the apparent partitioning rate constants $\left(\mathrm{k}_{1}{ }^{\prime} / \mathrm{k}_{-1}{ }^{\prime}\right.$ and $\mathrm{k}_{-3}{ }^{\prime} / \mathrm{k}_{3}{ }^{\prime}$, with $\mathrm{k}_{-1}{ }^{\prime}=\mathrm{k}_{3}{ }^{\prime}$ and $\left.\mathrm{k}_{-3}{ }^{\prime}=100 \times \mathrm{k}_{1}{ }^{\prime}\right)$ are given by the distribution of the compounds between the aqueous and the bilayer phases and can be calculated from the partition coefficients and the volume ratios of the aqueous and lipid phases. To vary the partition coefficient
$P$ in the simulations we changed either $\mathrm{k}_{-1}{ }^{\prime}$ at a constant $\mathrm{k}_{1}{ }^{\prime}$ or alternatively kept $\mathrm{k}_{-1}{ }^{\prime}$ constant and varied $\mathrm{k}_{1}{ }^{\prime}$.

Figure $8 \mathrm{a}$ shows the simulation results with the settings $\mathrm{k}_{1}{ }^{\prime}=1$ and $\mathrm{k}_{2}{ }^{\prime}$ between 0.001 and 100,000 . The partition coefficient $P$ was set to values between 0.1 and 1000 . The remaining apparent rate constants were calculated from these parameters as described under Material and Methods. The value $t_{90}$ gives the time point when $90 \%$ of the maximal luminal permeant concentration $\left[A_{\mathrm{ai}}\right]$ is reached. It is negatively correlated with the overall permeation rate. With $\mathrm{k}_{1}{ }^{\prime}=1$, the simulation revealed the following results. (1) If $\mathrm{k}_{2}{ }^{\prime} \leq \mathrm{k}_{-1}{ }^{\prime}$, i.e. if the flip flop rate constant is slower than the experimental (apparent) desorption rate constant, the permeation rate increases with increasing $\mathrm{k}_{2}{ }^{\prime}$. At a constant $\mathrm{k}_{2}{ }^{\prime}$ $\left(\leq \mathrm{k}_{-1}{ }^{\prime}\right)$, an increase in $P$, i.e. a decrease in $\mathrm{k}_{-1}{ }^{\prime}$, results in an increase of the permeation rate. Under these conditions low $P$ values are unfavourable for the permeation rate. Interestingly, above a certain threshold of $P$ (under the chosen conditions if $P \geq 100$, i.e. $\mathrm{k}_{-1}{ }^{\prime} \leq 10$ ), any further increase in $P$, i.e. decrease in $\mathrm{k}_{-1}{ }^{\prime}$, is without influence on the permeation rate. This is an important finding regarding the interpretation of our experimental data. (2) If $\mathrm{k}_{2}{ }^{\prime}>\mathrm{k}_{-1}{ }^{\prime}, t_{90}$ is independent of $\mathrm{k}_{2}{ }^{\prime}$. Under these conditions and at a constant $\mathrm{k}_{1}{ }^{\prime}$, the permeation rate is determined by $\mathrm{k}_{-1}{ }^{\prime}$ and $\mathrm{k}_{3}{ }^{\prime}$, and thus, by $P$. Surprisingly and in contrast to the findings with $\mathrm{k}_{2}{ }^{\prime} \leq \mathrm{k}_{-1}{ }^{\prime}$, high $P$ values result in a lower permeation rate, while low membrane affinity leads to faster permeation (all at a 
SA
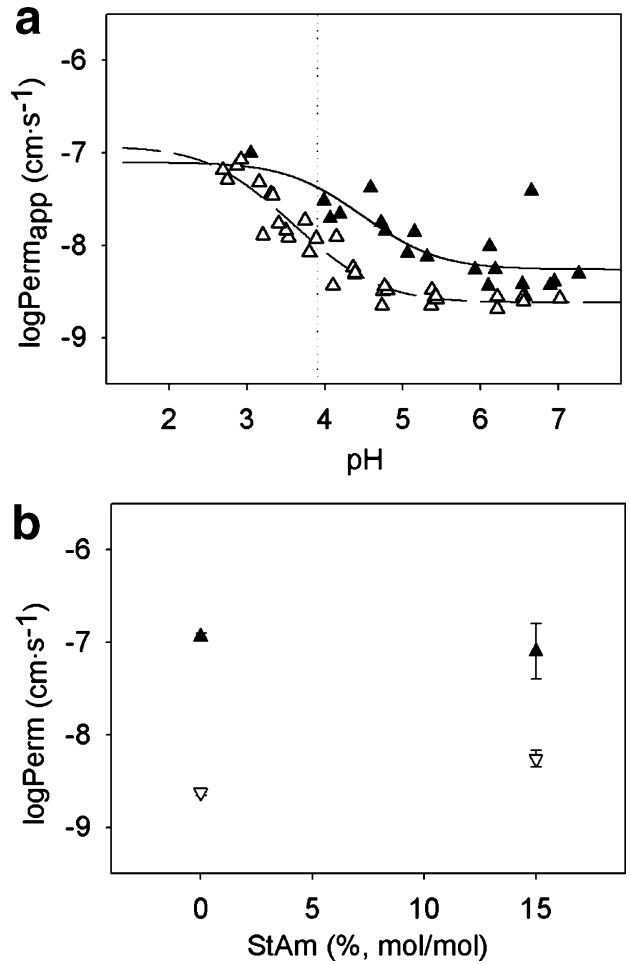

AA
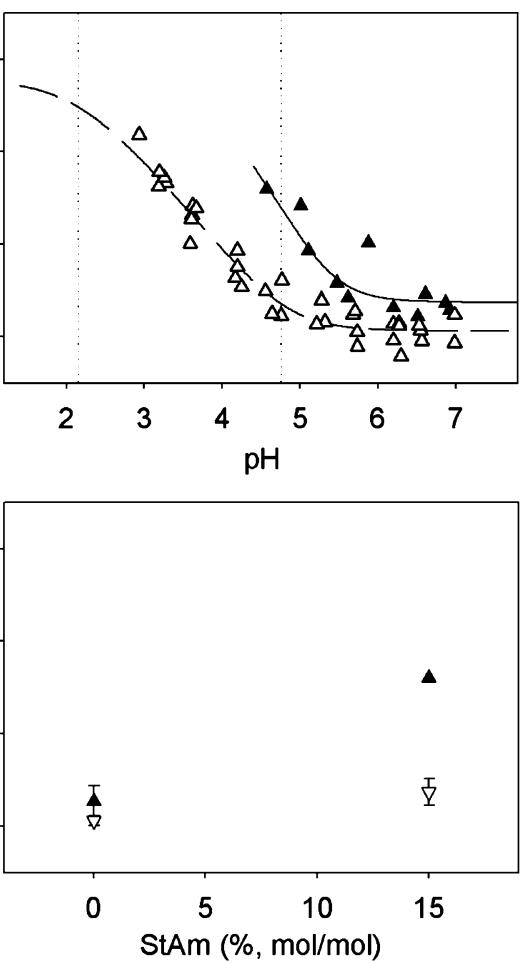

2-OH-NA
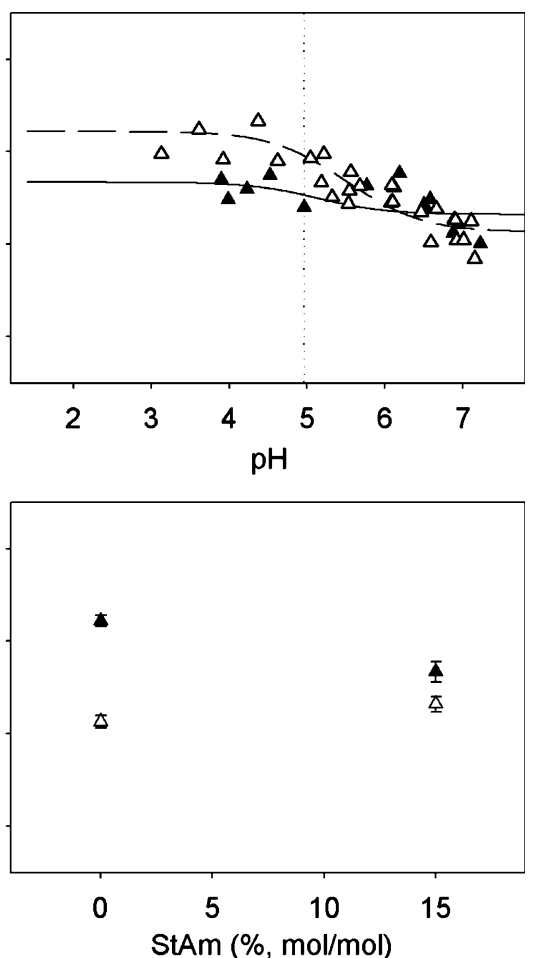

Fig. 7. Permeation across $\mathrm{PhC}$ and $\mathrm{PhC} / \mathrm{StAm}$ bilayers: a Membrane permeation of SA, AA and 2-OH-NA across $\mathrm{PhC}(\triangle)$ and $\mathrm{PhC} / \mathrm{StAm}$ $(85 / 15)(\mathbf{\Delta})$ bilayers. The $\log P e r m / p H$ profiles were fitted as described in Fig. 6 considering the permeation of all ionization species. b Fitted $\log$ Perm values for the different ionization species $\left(\log\right.$ erm $\left.^{A H} \boldsymbol{\Lambda} ; \log \mathrm{Perm}^{A^{-}} \nabla\right)$ in $\mathrm{PhC}$ and PhC/StAm 85/15 liposomes. Data are from three independent experiments.

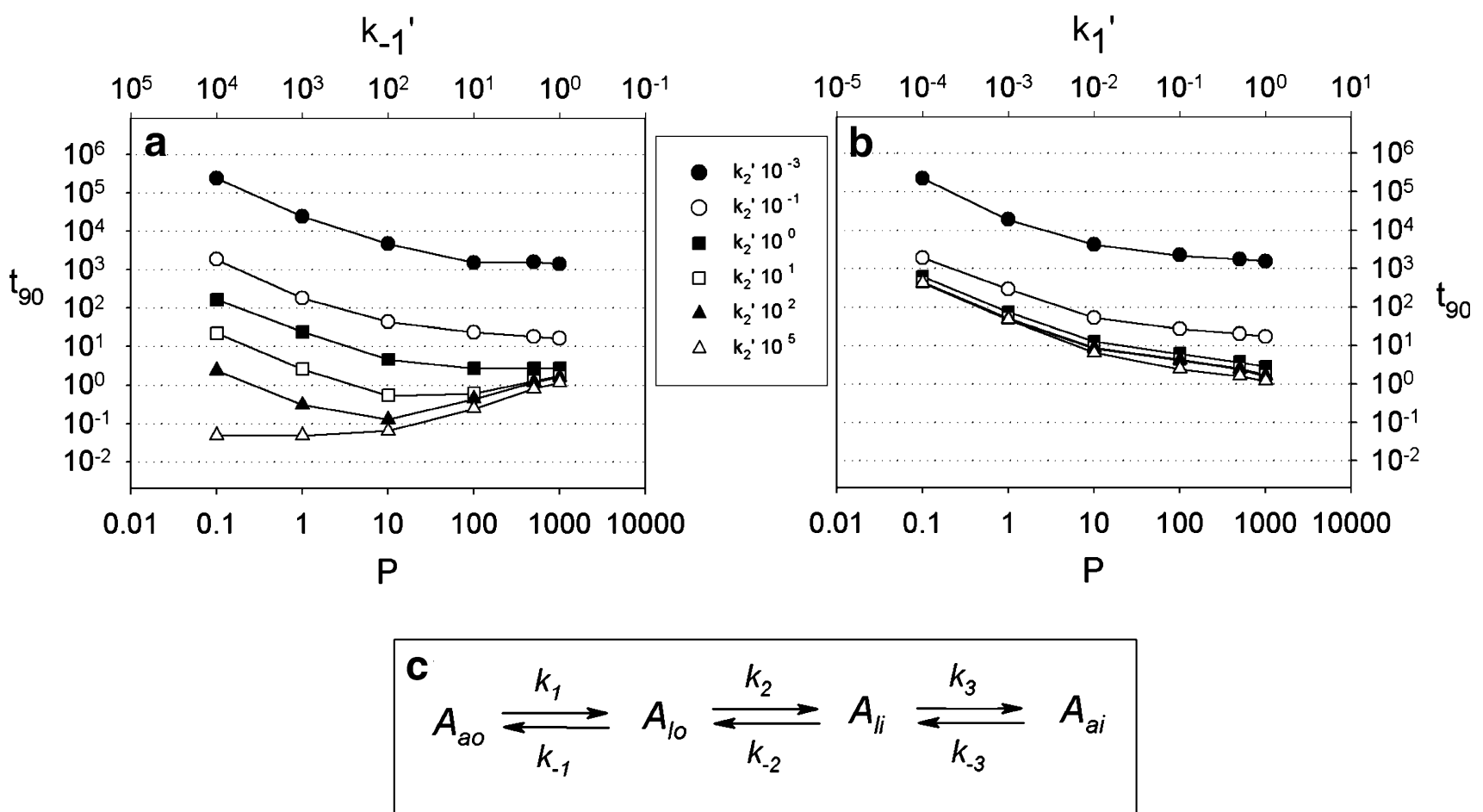

Fig. 8. Simulations of the permeation kinetics. a, b The time when $90 \%$ of the final permeant concentration in the liposomal lumen is reached was calculated by the numerical solution of the differential equations derived from the permeation model (c). Influence of $P$ and the translocation rate constant $\mathrm{k}_{2}{ }^{\prime}$ at a constant partition rate constant $\mathrm{k}_{1}{ }^{\prime}=1$ (a) and at a constant dissoziation rate constant $\mathrm{k}_{-1}{ }^{\prime}=1(\mathbf{b})$. 
constant $\left.\mathrm{k}_{1}{ }^{\prime}\right)$. Under these conditions $\mathrm{k}_{3}{ }^{\prime}$ is rate-limiting. At $P \leq 10$ (and $\mathrm{k}_{2}{ }^{\prime}>\mathrm{k}_{-1}{ }^{\prime}$ ), a further decrease in $P$ has no influence on the permeation rate.

The above simulations were performed with a constant $\mathrm{k}_{1}{ }^{\prime}$ and variations in $P$ were achieved by altering $\mathrm{k}_{-1}{ }^{\prime}$. Fig. $8 \mathrm{~b}$ shows the results with the settings $\mathrm{k}_{-1}{ }^{\prime}=1, \mathrm{k}_{2}^{\prime}$ between 0.001 and 100,000 and $P$ between 0.1 and 1,000. In this case alterations in $P$ were accomplished by varying $\mathrm{k}_{1}{ }^{\prime}$. For (1) $\mathrm{k}_{2}^{\prime} \leq \mathrm{k}_{-1}{ }^{\prime}$ the simulations revealed a similar picture as in Fig. 8a. The permeation rate is determined by $\mathrm{k}_{2}{ }^{\prime}$ and is positively correlated with $P$. In parallel to the findings described above with $\mathrm{k}_{1}{ }^{\prime}=1$, the influence of $P$ on the permeation rate is more pronounced if $P \leq 100$. (2) In contrast to the findings described for a constant $\mathrm{k}_{1}{ }^{\prime}=1$, the simulations with the settings used in Fig. 8b revealed a direct relationship between $P$ and the permeation rate also in the case $\mathrm{k}_{2}{ }^{\prime} \geq \mathrm{k}_{-1}{ }^{\prime}$.

To conclude, an increase in the membrane affinity may lead to an increase or a decrease in the permeation rate constant or may have no effect at all, depending on the ratios between the single rate constants of the partitioning and translocation processes as well as on the volume ratios of the membrane and the aqueous phases.

It should be mentioned here, that in symmetrical systems, i.e., equal volumes of the aqueous phases, $\mathrm{k}_{1}{ }^{\prime}$ is higher than $\mathrm{k}_{-1}{ }^{\prime}$ if $P>1$. The simulations need to be extended in this case, e.g., by our free online tool "PermSim" (http://www.biopharmacy.ethz.ch/simulations/PermSim).

\section{DISCUSSION}

The aim of this study was to explore the influence of Chol and two charged lipids on the $\mathrm{pH}$-dependent membrane affinity and membrane permeation of three test compounds and to investigate the relationship between the two processes.

Two well-defined and well-characterized liposomal test systems were used to study membrane affinity and permeation. Affinity was determined by equilibrium dialysis with radio-labeled compounds (22) and permeation was measured with the $\mathrm{Tb}(\mathrm{III})$-permeation assay $(14,19)$. We applied a linear first order differential system of equations to numerically describe the process of bilayer permeation based on the rate constants of partitioning and translocation. This provided revealing insight into the complex relationship between membrane affinity and permeation.

All partition and permeation profiles followed the Henderson-Hasselbalch function. The partition and permeation coefficients of the net neutral species were generally higher or equal to the partition or permeation coefficients, respectively, of the ionized species, except for the permeation of AA. The affinity and permeation of the anions were, however, high enough to control partitioning and permeation around the physiological $\mathrm{pH} 7$ in all systems. As shown with DCBA, permeation at $\mathrm{pH} 7$ was even in the absence of any intramolecular hydrogen bonding capacities anion-controlled. We are currently investigating this phenomenon with a larger series of structurally diverse aromatic carboxylic acids.

Lipid bilayer permeation of neutral and anionic SA has been studied in an excellent work with planar lipid bilayers more than thirty years ago by Gutknecht and Tosteson (9). The conclusion of this early work was different from ours, as the ratio between the calculated permeation coefficients of the neutral acid and the anion was magnitudes higher than the ratio we describe here and in our recent work (14). Plotting the early data of permeation (experimental flux / concentration) against the $\mathrm{pH}$, the shapes of the permeation profiles of Gutknecht and Tosteson and of our own work are notably similar, with the exception that the inflection point of the profile by Gutknecht and Tosteson is around $\mathrm{pH} 6$ instead of 2.75 , i.e. the $p K_{a}$ of SA. This could have different reasons, one of them is a pH shift at the membrane surface caused by the high amount of anions in the bilayer (see below) leading to a lower $\mathrm{pH}$ at the membrane surface than in the bulk solution (6). This surface-charge induced $\mathrm{pH}$ drop can explain part of the observed shift of the inflection point in the redrawn permeation $/ \mathrm{pH}$ profile. Permeation induced $\mathrm{pH}$ shifts as described by Antonenko et al. (27) can be ruled out as the concentrations of SA were equal on both sides of the membrane. The second possible reason for the shift in the inflection point is the unstirred water layer which forms an additional barrier for neutral and anionic SA permeation in the planar lipid bilayer system (28). Gutknecht and Tosteson got a perfect agreement between their data and a model including diffusion coefficients for $\mathrm{HA}$ and $\mathrm{A}^{-}$across the unstirred water layer. This led to a correction of the permeation coefficient of the neutral acid by a factor of about $10^{5}$. However, this factor would be significantly lower if the above mentioned $\mathrm{pH}$ shift at the membrane surface due to the high salicylate concentration within the bilayer would be taken into account.

It should in addition be kept in mind that the planar lipid bilayers of Gutknecht and Tosteson contained decane. The solvent could have a similar effect on the partitioning of salicylic acid and salicylate as n-octanol; the ratio between the two partition coefficients is higher in the n-octanol/buffer system than in the egg $\mathrm{PhC}$ liposomes (14).

Finally, and this is the point that should be carefully investigated, in the experiments by Gutknecht and Tosteson, measurements $\geq \mathrm{pH} 5$ were done at a total SA concentration of $0.1 \mathrm{M}$. Considering the partition coefficient of the anionic species in our liposomal system, i.e. 7, and a molar local lipid concentration of about $1 \mathrm{M}$ within the bilayer, this leads to permeant/lipid ratios in the range of $1 / 1$, which certainly affect the partitioning and permeation of any solute. At this high anion density within the membrane at $\mathrm{pH} \geq p K_{a}$, a reduction of anion partitioning as compared to ideal conditions, i.e., low permeant concentrations, can be expected due to electrostatic repulsion at the membrane surface (30). This reduction may result in a lower permeation coefficient of the anion than under ideal conditions.

This applies likewise to the experiments of Saparov et al. (29), which were performed to proof that we are measuring artefacts with our liposomal assay. The $10 \mathrm{mM}$ SA used by Saparov et al. leads to a similar lipid/permeant ratio of about $1 / 1$ at $\mathrm{pH}$ 3. This high ratio affects the membrane characteristics in general, and any $\mathrm{pH}$ shift measured by Saparov et al. at the membrane surface is not only due to the permeation of acid but also to the high charge density at the membrane surface $(6,30)$.

Our own system is free of solvents such as decane and contains $\geq 2,000$ times lower permeant concentrations than the system of Gutknecht and Tosteson and 20-2,000 times lower concentrations than used by Saparov et al. Under our 
experimental conditions, the permeation coefficients are concentration-independent (data not shown) and we neither observe a significant $\mathrm{pH}$ shift at the membrane surface by zetapotential measurements (14) nor by the addition of $\mathrm{pH}$ sensitive fluorescent lipid probes to the membrane (data not shown). We can exclude an unstirred water layer of the dimension of the planar lipid bilayer system. The average maximal distance of a solute to a lipid bilayer is about $200 \mathrm{~nm}$ while the unstirred water layer in the system of Gutknecht and Tosteson was estimated to be $0.13 \mathrm{~mm}$. We certainly have a clear advantage over other systems as we do not need to correct our experimental data for any changes in the surface $\mathrm{pH}$ caused by the permeant and for an unstirred water layer, respectively. This is reflected in the perfect agreement of the inflection points of the affinity and permeation/pH profiles with the $p K_{a}$ of the permeants (with the exception of the StAm-containing systems).

In this work, the lipid composition of the liposomes had a significant influence on the membrane affinity and permeation of the tested compounds (Table IV). Interestingly, the effects on the permeation rates were not in accordance with the effects on the membrane affinity. The tested lipids were $\mathrm{PhC}$, Chol, PhI and StAm. PhC is the main phospholipid in mammalian cell membranes and $\mathrm{PhC}$ liposomes were therefore used as the reference system.

Chol is found in biological membranes in variable amounts and leads to major changes in the structure and fluidity of the lipid bilayer $(17,31,32)$ which in turn influence the membrane affinity and permeation of solutes. Several studies (16,32-34) revealed a significant decrease of the membrane affinity of a variety of acidic, basic and neutral drugs when Chol was present in phospholipid bilayers. This was assigned to motion restrictions of the fatty acyl chain regions close to the lipid head groups and to the higher rigidity of the membrane induced by Chol in general, leading to an impeded integration of the drug molecules into the lipid bilayer. In this study, we found a significant decrease in membrane affinity of the net neutral and anionic species of SA and AA in presence of 25 and $40 \%$ Chol compared to $\mathrm{PhC}$ liposomes. The membrane affinities of the neutral and ionized species were affected in a similar manner, i.e. the effect of Chol was independent of the ionization state of the compound. This supports the hypothesis that the reduction of the membrane affinity is rather due to the lower flexibility of the lipid bilayer than to an influence in the head group region. The latter would probably affect electrostatic interactions and therefore have divergent effects on the affinity of neutral and ionized species.

In general, the membrane affinity is dependent on the ionization state of the solute and on the net charges of the membrane lipids. Positively charged propranolol and desipramine had a higher affinity to negatively charged than to net neutral membranes due to electrostatic attraction forces $(6,7)$. In the presence of the negatively charged $\mathrm{PhI}$, we found a major decrease in the membrane affinity of the negatively charged species of SA and AA, the affinity of the neutral species was less affected. These effects could be assigned to the electrostatic repulsion between the anionic species and the negatively charged membrane.

In contrast, $\log P^{A^{-}}$of SA and AA were considerably increased in the presence of $15 \%$ StAm while $\log P^{A H}$

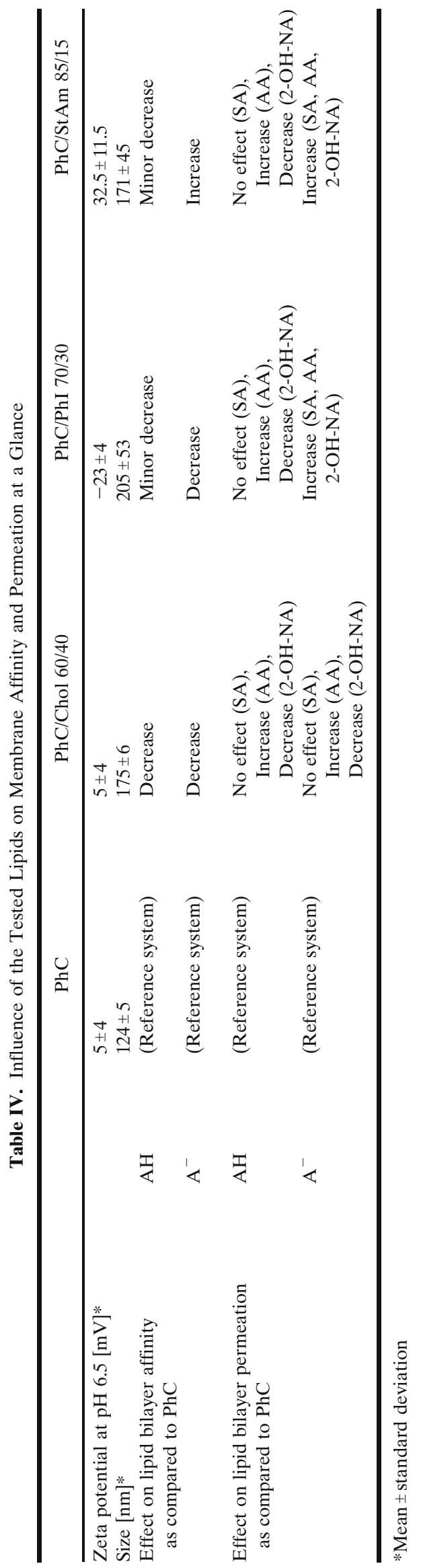


remained unchanged or was slightly decreased. The increase in $\log P^{A^{-}}$is probably due to the electrostatic attractions between the positively charged membrane and the negatively charged solute. As for Chol, the influence of the charged lipids $\mathrm{PhI}$ and StAm on the membrane affinity was consistent for SA and AA. From these results and the cited partition studies we conclude that the influence of particular lipids on the membrane affinity of acids and bases is predictable in a qualitative manner considering the rigidity of the bilayer and the electrostatic interactions between the charged solutes and the lipid headgroups.

Membrane permeation did not follow such clear rules. In contrast to the findings in the partition experiments, changes in the lipid composition had unpredictable effects on membrane permeation. There was no clear correlation between membrane affinity and permeation.

It was reported that solute permeation is reduced in Chol-containing membranes. De Gier et al. (35) observed decreased permeation coefficients of glycerol and erythritol with 10 to $50 \%$ Chol in egg PhC bilayers. Frezard et al. (5) described decreased permeability coefficients of anthracyclines with increasing amounts of Chol between 20 and $45 \%$ in egg PhC/egg phosphatidic acid membranes at $\mathrm{pH} 6$.

In our study the influence of $\mathrm{Chol}$ on the permeation of the tested compounds was not consistent. The membrane permeation of SA was not influenced by 25 and $40 \%$ Chol, despite the significant decrease in membrane affinity at these Chol contents. Except at $10 \%$ Chol, we observed a decrease in the permeation coefficients of 2-OH-NA with all tested Chol concentrations. This effect was independent of the ionization state of the solute and thus probably due to a general influence of $\mathrm{Chol}$ on the rigidity of the membrane. The different behaviors of SA and 2-OH-NA are coherent with the simulation results. Under the condition that the apparent translocation rate constant is slower than the apparent membrane dissociation rate constant $\left(\mathrm{k}_{2}^{\prime}<\mathrm{k}_{-1}{ }^{\prime}\right)$, $P$ values above a certain threshold, which depends on the single apparent rate constants, have no influence on the permeation rate while $P$ values below this threshold are positively correlated with the permeation rate.

The effects of the charged lipids on the membrane affinity and permeation of the test compounds are further emphasizing the intriguing relationship between membrane affinity and permeation. As expected from the possible electrostatic interactions, the negatively charged $\mathrm{PhI}$ reduced the membrane affinity of the anions of SA and AA while the positively charged StAm led to a significant increase. Surprisingly, the permeation of the anions was increased in presence of both $\mathrm{PhI}$ and StAm, respectively. Although the permeation-enhancing effect of $\mathrm{PhI}$ would not be expected from the membrane affinity measurements, the experimental results can be simulated by varying the rate constants of partitioning and translocation.

The net neutral AA shows a striking permeation behavior. In $\mathrm{PhC}$ and in the presence of $25 \%$ Chol it permeated about 300 times slower than the cation and at a similar rate as its corresponding anion. This might be due to strong electrostatic attraction forces between the ampholytic AA and the lipid headgroups lowering the chance for a flip-flop event. The addition of other lipids (except 25\% Chol) beside $\mathrm{PhC}$ led to an increase of the permeation coefficient of the net neutral
AA. This permeation enhancing effect of Chol, PhI or StAm could be related to an interruption of the electrostatic attractions between phospatidylcholine head groups and net neutral AA (36).

Investigating the influence of Chol on the membrane permeation, we found a discontinuous behavior at $10 \%$ with all tested compounds. This is in line with the exceptional membrane behavior at low Chol concentrations observed before (37-39) and could be related to the peculiarities in the phase diagrams of $\mathrm{PhC/Chol} \mathrm{mixtures} \mathrm{as} \mathrm{suggested} \mathrm{by}$ Mouritsen et al. (17). These authors described increased binding of ethanol to dimyristoylphosphatidylcholine bilayers with $4 \%$ Chol while $35 \%$ Chol had the opposite effect. Additionally they observed that low levels (5\%) of Chol increase the ${ }^{22} \mathrm{Na}$ flux across dipalmitoylphosphatidylcholine bilayers, while the effect was inverse with $40 \%$ Chol. The phase diagram of binary mixtures of $\mathrm{PhC} / \mathrm{Chol}$ shows a depression of the chain melting temperature and a narrow coexistence region of solid-ordered and liquid-disordered state up to $10 \%$ Chol. With higher amounts of Chol the socalled liquid-ordered state is formed (40). Thus, the exceptional behavior found for the permeation profiles of $\mathrm{SA}$ and $\mathrm{AA}$ at $10 \%$ Chol is likely to be due to the structural characteristics of the Chol-containing membrane. The membrane is affected in a different manner by low Chol concentrations than by high Chol concentrations.

The addition of StAm to the bilayer led to significant deviations of the inflection points of the $\log D / \mathrm{pH}$ profiles (SA, AA) and of the $\log$ Perm $_{\mathrm{app}} / \mathrm{pH}$ profile (AA) from the titrated $p K_{a}$ values. The dissociation constant of StAm in our liposomal systems was estimated by zeta potential measurement of the liposomes revealing an apparent $\mathrm{pK}_{\mathrm{a}}$ of $\sim 9.7$ (data not shown). This is in agreement with the findings of Ptak et al. (41) who determined the $p K_{a} \sim 9.5$ in $\mathrm{PhC}$ liposomes by ${ }^{13} \mathrm{C}$ NMR spectroscopy. Thus, it can be excluded that the dissociation equilibrium of the amine group interferes with the experimental data. The membrane is positively charged within the investigated $\mathrm{pH}$ range. The shift of the inflection point to higher values could be due to the formation of micelles by StAm and the permeant in the aqueous phase. Size distribution measurements, however, did not reveal the presence of micelles.

Beside the influence of the single rate constants on the permeation kinetics, the simulations also revealed the high impact of the volume ratios on the final permeation coefficients. The volume ratios even predefine the rate limiting step(s). The permeation rates as well as the effects of experimental conditions can therefore completely change from one system to another. This should be kept in mind when comparing results from different systems such as liposomes and planar lipid bilayers. The ratio of lipid to aqueous volumes is by magnitudes higher in the liposomal system than in the planar lipid bilayer system. The apparent rate constants are directly dependent on these volume ratios. It may therefore happen that permeation of a particular compound is determined by the partition rate constants in one system but by the translocation rate constant(s) in the other system, depending on the volume ratios of the aqueous and membrane phases. Membrane affinity or $\log P_{\text {Octanol }}$ may thus correlate well with membrane permeation in one system but not in the other. The single rate constants of the 
permeation process become however computable by changing the volume ratios in the permeation experiments.

In conclusion, membrane affinity and membrane permeation are dependent on the membrane composition. The influence of single lipids on the membrane affinity of the test compounds followed straightforward rules considering bilayer rigidity and electrostatic interactions. Bilayer permeation on the other hand appeared more complex. There was no direct relationship between the effects of the membrane composition on membrane affinity and permeation. It was found that the lipid composition can even have opposite effects on compound partitioning and permeation. As could be shown by simulations the permeation may positively, negatively or not correlate at all with the membrane affinity, depending on the interplay of the rate constants of partitioning and flip-flop and of the volume ratios of aqueous and lipid phases.

\section{ACKNOWLEDGEMENT}

We would like to thank Maja Günthert for experimental support as well as Knud Zabrocki and Marian Brandau (Martin-Luther-University Halle, Germany) for their advice concerning the mathematical solution of the differential equation system.

\section{REFERENCES}

1. G. D. Eytan. Mechanism of multidrug resistance in relation to passive membrane permeation. Biomed. Pharmacother. 59:90-97 (2005).

2. S. D. Krämer. Lipid bilayers in ADME: Permeation barriers and distribution compartments. In B. Testa, S. D. Krämer, H. Wunderli-Allenspach, and G. Folkers (eds.), Pharmacokinetic Profiling in Drug Research: Biological, Physicochemical and Computational Strategies, Wiley-VCH, Weinheim, 2005.

3. P. Lauger, R. Benz, G. Stark, E. Bamberg, P. C. Jordan, A. Fahr, and W. Brock. Relaxation studies of ion transport systems in lipid bilayer membranes. O. Rev. Biophys. 14:513-598 (1981).

4. P. T. Mayer, T.-X. Xiang, R. Niemi, and B. D. Anderson. A hydrophobicity scale for the lipid bilayer barrier domain from peptide permeabilities: nonadditivities in residue contributions. Biochemistry 42:1624-1636 (2003).

5. F. Frézard and A. Garnier-Suillerot. Permeability of lipid bilayer to anthracycline derivatives. Role of the bilayer composition and of the temperature. Biochim. Biophys. Acta 1389:1322 (1998).

6. S. D. Krämer, A. Braun, C. Jakits-Deiser, and H. WunderliAllenspach. Towards the predictability of drug-lipid membrane interactions: the pH-dependent affinity of propanolol to phosphatidylinositol containing liposomes. Pharm. Res. 15:739-744 (1998).

7. M. Marenchino, A. L. Alpstäg-Wöhrle, B. Christen, H. WunderliAllenspach, and S. D. Krämer. [alpha]-tocopherol influences the lipid membrane affinity of desipramine in a $\mathrm{pH}$-dependent manner. Eur. J. Pharm. Sci. 21:313-321 (2004).

8. S. D. Krämer, C. Jakits-Deiser, and H. Wunderli-Allenspach. Free fatty acids cause $\mathrm{pH}$-dependent changes in drug-lipid membrane interactions around physiological pH. Pharm. Res. 14:827-832 (1997).

9. J. Gutknecht and D. C. Tosteson. Diffusion of weak acids through lipid bilayer membranes. Role of chemical reactions in the unstirred layer. Science 182:1258-1261 (1982).

10. P. A. Shore, B. B. Brodie, and C. A. Hogben. The gastric secretion of drugs: a pH partition hypothesis. J. Pharmacol. Exp. Ther. 119:361-369 (1957).
11. T.-X. Xiang and B. D. Anderson. Substituent contributions to the transport of substituted p-toluic acids across lipid bilayer membranes. J. Pharm. Sci. 83:1511-1518 (1994).

12. K. A. Youdim, A. Avdeef, and N. J. Abbott. In vitro transmonolayer permeability calculations: often forgotten assumptions. Drug Discov. Today 8:997-1003 (2003).

13. L. X. Yu, E. Lipka, J. R. Crison, and G. L. Amidon. Transport approaches to the biopharmaceutical design of oral drug delivery systems: prediction of intestinal absorption. Adv. Drug Deliv. Rev. 19:359-376 (1996).

14. A. V. Thomae, H. Wunderli-Allenspach, and S. D. Krämer. Permeation of aromatic carboxylic acids across lipid bilayers: the pH-partition hypothesis revisited. Biophys. J. 89:1802-1811 (2005).

15. J. H. Ipsen, G. Karlstrom, O. G. Mouritsen, H. Wennerstrom, and M. J. Zuckermann. Phase equilibria in the phosphatidylcholine-cholesterol system. Biochim. Biophys. Acta 905:162-172 (1987).

16. D. Kurad, G. Jeschke, and D. Marsh. Lateral ordering of lipid chains in cholesterol-containing membranes: high-field spinlabel EPR. Biophys. J. 86:264-271 (2004).

17. O. G. Mouritsen and M. J. Zuckermann. What's so special about Cholesterol? Lipids 39:1101-1113 (2004).

18. M. J. Hope, M. B. Bally, G. Webb, and P. R. Cullis. Production of large unilamellar vesicles by a rapid extrusion procedure. Characterization of size distribution, trapped volume and ability to maintain a membrane potential. Biochim. Biophys. Acta 812:55-65 (1985).

19. S. D. Krämer and H. Wunderli-Allenspach. No entry for TAT(44-57) into liposomes and intact MDCK cells: novel approach to study membrane permeation of cell-penetrating peptides. Biochim. Biophys. Acta 1609:161-169 (2003).

20. S. D. Krämer, J. A. Hurley, D. J. Begley, and N. J. Abbott Lipids in blood-brain barrier models in vitro I: TLC and HPLC for the analysis of lipid classes and long polyunsaturated fatty acids. In Vitro Cell. Dev. Biol., Anim. 38:557-565 (2002).

21. R. Singh, M. Ajagbe, S. Bhamidipati, Z. Ahmad, and I. Ahmad. A rapid isocratic high-performance liquid chromatography method for determination of cholesterol and 1,2-dioleoyl-snglycero-3-phosphocholine in liposome-based drug formulations. J. Chromatogr., A 1073:347-353 (2005).

22. G. M. Pauletti and H. Wunderli-Allenspach. Partition coefficients in vitro: artificial membranes as a standardized distribution model. Eur. J. Pharm. Sci. 1:273-282 (1994).

23. A. Martin, P. Bustamante, and A. H. C. Chun. Physical Pharmacy, Lea \& Febiger, Philadelphia, 1993.

24. B. W. Kernighan and D. M. Ritchie. The C Programming Language, Prentice Hall, New Jersey, 1988.

25. C. Huang and J. T. Mason. Geometric packing constraints in egg phosphatidylcholine vesicles. Proc. Nat'l. Acad. Sci. USA. 75:308-310 (1978).

26. M. Trumbore, D. W. Chester, J. Moring, D. Rhodes, and L. G. Herbette. Structure and location of amiodarone in a membrane bilayer as determined by molecular mechanics and quantitative x-ray diffraction. Biophys. J. 54:535-543 (1988).

27. Y. N. Antonenko, G. A. Denisov, and P. Pohl. Weak acid transport across bilayer lipid membrane in the presence of buffers. Theoretical and experimental $\mathrm{pH}$ profiles in the unstirred layers. Biophys. J. 64:1701-1710 (1993).

28. A. Avdeef, P. Artursson, S. Neuhoff, L. Lazorova, J. Grasjo, and S. Tavelin. Caco-2 permeability of weakly basic drugs predicted with the double-sink PAMPA pKa(flux) method. Eur. J. Pharm. Sci. 24:333-349 (2005).

29. S. M. Saparov, Y. N. Antonenko, and P. Pohl. A new model of weak acid permeation through membranes revisited: does Overton still rule? Biophys. J. 90:L86-L88 (2006).

30. S. D. Krämer. Liposome/water partitioning: theory, techniques and applications. In B. Testa, G. Folkers, and R. Guy (eds.), Pharmacokinetic Profiling in Drug Research: Biological, Physicochemical and Computational Strategies, Wiley-VCH, Weinheim, 2001.

31. A. Kusumi, W. K. Subczynski, M. Pasenkiewicz-Gierula, J. S. Hyde, and H. Merkle. Spin-label studies on phosphatidylcholinecholesterol membranes: effects of alkyl chain length and unsatu- 
ration in the fluid phase. Biochim. Biophys. Acta 854:307317 (1986).

32. J. A. Urbina, S. Pekerar, H. B. Le, J. Patterson, B. Montez, and E. Oldfield. Molecular order and dynamics of phosphatidylcholine bilayer membranes in the presence of cholesterol, ergosterol and lanosterol: a comparative study using $2 \mathrm{H}-, 13 \mathrm{C}-$ and $31 \mathrm{P}-$ NMR spectroscopy. Biochim. Biophys. Acta 1238:163-176 (1995).

33. J. R. Henriksen, A. C. Rowat, E. Brief, Y.-W. Hsueh, J. L. Thewalt, M. J. Zuckermann, and J. H. Ipsen. Universal behaviour of membranes with sterols. Biophys. J. 90:1639-1649 (2005).

34. C. Lagerquist, F. Beigi, A. Karlen, H. Lennernas, and P. Lundahl. Effects of cholesterol and model transmembrane proteins on drug partitioning into lipid bilayers as analysed by immobilized-liposome chromatography. J. Pharm. Pharmacol. 53:1477-1487 (2001).

35. J. De Gier, J. G. Mandersloot, and L. L. M. Van Deenen. Lipid composition and permeability of liposomes. Biochim. Biophys. Acta 150:666-675 (1968).

36. P. L. Yeagle, W. C. Hutton, C. Huang, and R. B. Martin. Phos- pholipid head-group conformations; intermolecular interactions and cholesterol effects. Biochem. 16:4344-4349 (1977).

37. E. Corvera, O. G. Mouritsen, M. A. Singer, and M. J. Zuckermann. The permeability and the effect of acyl-chain length for phospholipid bilayers containing cholesterol: theory and experiment. Biochim. Biophys. Acta 1107:261-270 (1992).

38. O. G. Mouritsen and K. Jorgensen. Dynamical order and disorder in lipid bilayers. Chem. Phys. Lipids 73:3-25 (1994).

39. T. Parasassi, A. M. Giusti, M. Raimondi, and E. Gratton. Abrupt modifications of phospholipid bilayer properties at critical cholesterol concentrations. Biophys. J. 68:1895-1902 (1995).

40. L. Miao, M. Nielsen, J. Thewalt, J. H. Ipsen, M. Bloom, M. J. Zuckermann, and O. G. Mouritsen. From lanosterol to cholesterol: structural evolution and differential effects on lipid bilayers. Biophys. J. 82:1429-1444 (2002).

41. M. Ptak, M. Egret-Charlier, A. Sanson, and O. Bouloussa. A NMR study of the ionization of fatty acids, fatty amines and Nacylamino acids incorporated in phosphatidylcholine vesicles. Biochim. Biophys. Acta 600:387-397 (1980). 